\title{
A novel and specific regulator of neuronal V-ATPase in Drosophila
}

$6{ }^{1}$ Genes Circuits Rhythms and Neuropathology, Brain Plasticity Unit, CNRS, ESPCI Paris, PSL

7 Research University, 10 rue Vauquelin, F-75005 Paris, France

8 2Dipartimento di Scienze Agroalimentari, Ambientali e Animali, University of Udine, Udine, Italy

9 and Université de Picardie Jules Verne, CNRS-UMR 7058 "Ecologie et Dynamique des Systèmes

10 Anthropisés" (EDYSAN), Amiens, France

$11{ }^{3} \mathrm{INCIA}$ - Institut de Neurosciences Cognitives et Intégratives d'Aquitaine, CNRS, Université de

12 Bordeaux, PAC Talence, allée Geoffroy Saint-Hilaire, F-33615 Pessac cedex, France

15 *Author for correspondence: serge.birman@espci.fr

18 "Present address: Sussex Neuroscience, School of Life Sciences, University of Sussex, Biology Road,

19 Brighton BN1 9QG, UK

20 §Present address: Neurodevelopment Laboratory, School of Biosciences, University of Birmingham,

21 Birmingham B15 2TT, UK 


\section{Abstract}

23 The V-ATPase is a highly conserved enzymatic complex that ensures appropriate levels of

24 organelle acidification in virtually all eukaryotic cells. While the general mechanisms of this

25 proton pump have been well studied, little is known about the specific regulations of neuronal

26 V-ATPase. Here, we studied CG31030, a previously uncharacterized Drosophila protein

27 predicted from its sequence homology to be part of the V-ATPase family. We found that this

28 protein is essential and apparently specifically expressed in neurons, where it is addressed to

29 synaptic terminals. We observed that CG31030 co-immunoprecipitated with V-ATPase subunits,

30 in particular with ATP6AP2, and that synaptic vesicles of larval motoneurons were not properly

31 acidified in CG31030 knockdown context. This defect was associated with a decrease in quantal

32 size at the neuromuscular junction, severe locomotor impairments and shortened lifespan.

33 Overall, our data provide evidence that CG31030 is a specific regulator of neuronal V-ATPase

34 that is required for synaptic vesicle acidification and neurotransmitter release.

36 Key words

37 CG31030/VhaAC45L, neuronal V-ATPase, synaptic vesicle acidification, quantal size,

\section{Drosophila melanogaster}




\section{Introduction}

44 Many cellular processes require a specific electrochemical environment for proper functioning,

45 such as post-translational modifications of proteins in the Golgi apparatus, lysosomal

46 degradation, endosomal ligand-receptor dissociation, or hormone concentration (reviewed in

47 Forgac, 2007). Eukaryotic cells use a highly conserved proton pump, called the vacuolar $\mathrm{H}^{+}-$

48 ATPase (V-ATPase), to achieve the adequate level of acidity in different cellular compartments

49 (Saroussi and Nelson, 2009). This large enzymatic complex must be tightly regulated, as it is

50 essential for it to be localized on the right membrane, and to fit the different $\mathrm{pH}$ ranges specific

51 to each organelle and cell type.

52 In neurons, the V-ATPase plays a crucial role at the synapse, being responsible for acidifying

53 synaptic vesicles and thus providing the driving force for neurotransmitter loading (Moriyama et

54 al., 1992). Recently, neuronal V-ATPase has also gained interest in the context of aging and

55 neurodegenerative diseases, as its dysregulation, and resulting impairment of the autophagy-

56 lysosomal pathway, have been linked to several pathologies such as Alzheimer and Parkinson

57 disease (Colacurcio and Nixon, 2016; Collins and Forgac, 2020). If the core mechanism of the

58 proton pump is now well understood, the regulations conferring the cell-specific functions of

59 neuronal V-ATPase remain largely unknown, considerably limiting its potential use as a

60 therapeutic target.

61 The V-ATPase complex is composed of a cytoplasmic domain $\left(\mathrm{V}_{1}\right)$ and a membrane-bound

62 domain $\left(\mathrm{V}_{0}\right)$. The $\mathrm{V}_{1}$ domain contains the catalytic unit responsible for ATP hydrolysis. The

63 energy resulting from this reaction powers a rotational molecular motor spanning from $V_{1}$ to $V_{0}$,

64 allowing protons to cross membranes through the port contained in $\mathrm{V}_{0}$ (Vasanthakumar and 
65 Rubinstein, 2020). The assembly of $V_{1}$ to $V_{0}$ is necessary for the pump to function, and reversible

66 dissociation of the two domains has been shown to occur as a way to regulate V-ATPase activity

67 (Collins and Forgac, 2020). Though the core mechanism stays the same, one V-ATPase can differ

68 from another by its composition. In vertebrates, as well as in Drosophila, $\mathrm{V}_{0}$ is made of five

69 subunits $\left(a, c, c^{\prime \prime}, d\right.$ and $e$ ), while $V_{1}$ contains eight subunits $(A, B, C, D, E, F, G$ and $H$ ) (Allan et al.,

70 2005). Each subunit can have several paralogs encoded by different genes, and each gene can

71 produce several isoforms, allowing many different possible combinations to form the full V-

72 ATPase complex. These differences of composition can also have regulatory effects on the

73 complex, both on its localization and on its functional properties (Vasanthakumar and

74 Rubinstein, 2020).

75 The V-ATPase can also be regulated by two accessory subunits, ATP6AP1/Ac45 and

76 ATP6AP2/PRR (Jansen and Martens, 2012). Both proteins are found in the nervous tissue but

77 are also required in other organs, and their mutation has been linked to cognitive impairments

78 as well as systemic symptoms like immunodeficiency or hepatopathy (Jansen et al., 2016;

79 Cannata et al., 2018). These two subunits interact directly with the $\mathrm{V}_{0}$ domain and are believed

80 to promote assembly of the membrane and soluble regions of the V-ATPase complex (Abbas et

81 al., 2020). Another potential accessory subunit exists in vertebrates, named ATP6AP1-like

82 (ATP6AP1L), which is homologous to ATP6AP1 and has not yet been functionally characterized.

83 Drosophila possesses identified homologs of ATP6AP1/Ac45 and ATP6AP2/PRR, named

84 VhaAC45 and ATP6AP2, respectively. These proteins also seem to contribute to assembly of the

85 V-ATPase in fly tissues (Schoonderwoert and Martens, 2002a; Guida et al., 2018). 
86 In this study, we examine the localization and function of CG31030, a novel ATP6AP1 homolog

87 in Drosophila whose unique characteristic is to be expressed selectively and ubiquitously in

88 neurons. Whereas a complete deficiency of this protein is lethal, we found that partial CG31030

89 knockdown in larval motoneurons impaired synaptic vesicle acidification, reduced quantal size,

90 which is the amplitude of the postsynaptic response to the release of a single synaptic vesicle,

91 and induced severe locomotion defects. We also report that CG31030 from brain tissue co-

92 immunoprecipitated with V-ATPase subunits of the $\mathrm{V}_{0}$ domain. Overall, our results indicate that

93 CG31030 is a novel accessory subunit of the neuronal V-ATPase that appears to be involved in

94 the regulation of synaptic activity.

95

96 Results

97 CG31030 is specifically expressed in neurons and addressed to synaptic areas

98 Here we studied in Drosophila the gene CG31030, identified in FlyBase as a paralog of VhaAC45/

99 CG8029, and as an ortholog of vertebrate ATP6AP1/AC45 (Thurmond et al., 2019). CG31030 and

100 VhaAC45 indeed share 69.9\% similarity in amino-acid sequences (Supplementary Figure 1A).

101 CG31030 is also classified in the V-ATPase family group by the InterPro database (accession:

102 Q8IMJO_DROME) (Mitchell et al., 2019). According to FlyAtlas (Chintapalli et al., 2007), VhaAC45

103 is expressed ubiquitously in Drosophila tissues. In contrast and interestingly, CG31030 seems to

104 be specifically expressed in the nervous system in both larval and adult flies, making it a possible

105 candidate for the specific regulation of neuronal V-ATPase (Supplementary Figure 1B). To

106 confirm this prediction, we checked by RT-qPCR the repartition of CG31030 transcripts in three

107 parts of the adult fly body: the head and thorax, which contains the brain and ventral nerve cord 
108 (VNC), respectively, and the abdomen, which is relatively poor in nervous tissue. Both females

109 and males showed highest expression in the head, minor expression in the thorax and no

110 detectable expression in the abdomen (Figure 1A). The expression of CG31030 therefore closely

111 follows the repartition of the nervous system.

112 The single-cell RNA-Seq Scope database (Davie et al., 2018) furthermore indicated that, in

113 Drosophila, CG31030 is expressed in all neurons, with few or no expression in glial cells. To

114 verify this, we expressed three different CG31030 RNAi either with either the pan-neuronal

115 driver elav-Gal4 or the pan-glial driver repo-Gal4 (see Supplementary Table 3 for genotypes of

116 the different Drosophila lines used in this study). The expression of CG31030 RNAi1 and

117 CG31030 $0^{\text {RNAi2 }}$ was found to be lethal at embryonic and $1^{\text {st }}$ larval stages, respectively, while

$118 \mathrm{CG} 31030^{\mathrm{RNAi3}}$ produced viable adults with a shortened longevity (Supplementary Figure 2), and

119 obvious locomotor impairments. This difference in phenotypes observed with different RNAi

120 constructs could be attributed to a variation in residual levels of the CG31030 protein. RT-qPCR

121 experiments showed that the pan-neuronal expression of CG31030 ${ }^{\mathrm{RNAi}}$ together with the RNAi

122 booster Dicer-2 (Dcr-2) was sufficient to decrease by more than $80 \%$ CG31030 transcripts

123 abundance in extracts from the adult heads (Figure 1B). On the other hand, glial expression of

124 this RNAi construct with repo-gal4 had no significant effects on CG31030 transcript level (Figure

125 1B), confirming that this gene is selectively expressed in neurons. It is interesting to note that

126 both $C G 31030^{\text {RNAi1 }}$ and $C G 31030^{\text {RNAi2 }}$ also induced lethal phenotypes at various developmental

127 stages when expressed either with a glutamatergic (VGlut-Gal4) or a cholinergic (Cha-Gal4)

128 neuronal driver (data not shown), in accordance with the RNA-Seq data of the Scope website

129 suggesting a pan-neuronal expression of this gene. 
130 To validate these observations, we placed the available MIMIC line CG31030 MI107 that contains a

131 stop codon inserted in the middle of the gene (Nagarkar-Jaiswal et al., 2015) over the deficiency

$132 D f(3 R)$ Exel6214 encompassing CG31030 (Parks et al., 2004). The resulting mutant, likely to be a

133 null, was found to be embryonic lethal, in agreement with the results obtained with two

134 CG31030 RNAi lines. Remarkably, re-expressing the gene selectively in neurons, using a UAS-

135 CG31030 construct driven by elav-Gal4, was sufficient to rescue this lethality, producing viable

136 and fertile adults with no obvious behavioral defects (Supplementary Table 1). These results

137 strongly indicate that CG31030 is an essential gene whose expression appears to be specifically

138 required in neurons.

139 Next, we studied the cellular localization of this protein. We used the CRISPR-Cas9 technique to

140 insert a small V5 epitope tag in frame at the $3^{\prime}$ end of its gene, disrupting the stop codon, thus

141 generating the $C G 31030^{\mathrm{V} 5}$ mutant line (Figure 1C). By immunostaining with a V5-specific

142 antibody, we observed that the general expression pattern of CG31030 in adult brain (Figure

143 1D) and larval CNS (Figure 1E) was widespread and quite similar to that of the synaptic marker

144 Cadherin-N (CadN), indicative of a predominantly synaptic localization. No specific signal was

145 detected in a control $w^{1118}$ line that does not contain the V5-tagged protein (not shown). Some

146 neuronal cell bodies were also marked with the V5 antibody in the CG31030 V5 line, both in adult

147 and larva, and the synapse-containing neuropile areas (like the antennal lobes) were not as

148 sharply defined as with the CadN antibody, suggesting that axons could also be immunopositive.

149 Finally, co-immunostaining of larval body muscles wall with anti-horseradish peroxidase (HRP)

150 antibodies, a marker of Drosophila neurons (Jan and Jan, 1982), showed precise co-localization 
bioRxiv preprint doi: https://doi.org/10.1101/2021.02.08.430253; this version posted April 21, 2021. The copyright holder for this preprint (which

was not certified by peer review) is the author/funder, who has granted bioRxiv a license to display the preprint in perpetuity. It is made available under aCC-BY 4.0 International license.

151 with the V5 signal, indicating that CG31030 is addressed to synaptic boutons at the larval

152 neuromuscular junction (Figure 1F).

153

154

155 


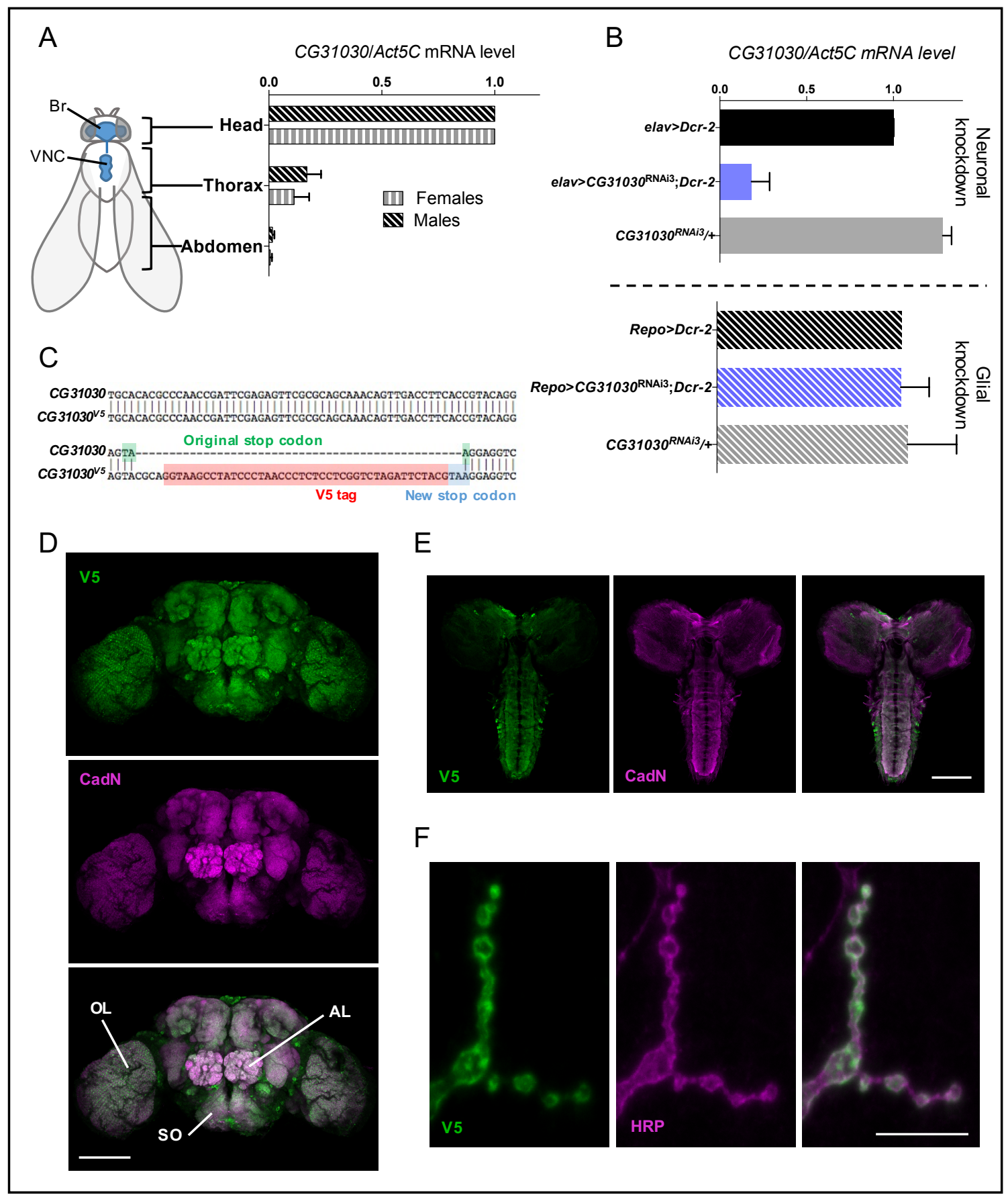

157 Figure 1. CG31030 is expressed in neurons and addressed to synaptic areas. (A) In both males and

158 females, CG31030 mRNA abundance follows the localization of the CNS (shown in blue on the fly sketch),

159 with the highest expression in the head. Br: brain, VNC: ventral nerve cord. Results of three independent

160 experiments. (B) Expression of $C G 31030^{\mathrm{RNAi} 3}$ with Dcr-2 in all neurons using elav-Gal4 decreased

161 CG31030 mRNA level in head by more than $80 \%$, while expression of the RNAi construct and Dcr- 2 in all 
162 adult glial cells with repo-Gal4 had not effect. Results of three independent experiments. Mean values

163 with SD are reported on the graphs. (C) Construction of the CG31030 V5 mutant strain. A V5 tag (in red)

164 was fused to the C-terminal end of the CG31030 protein by inserting the V5 coding sequence ended by a

165 new stop codon (in blue) in place of the original stop codon (in green) in the CG31030 gene using the

166 CRISPR-Cas9 technology. (D, E) Anti-V5 immunostaining in the CG31030 ${ }^{\mathrm{V} 5}$ strain revealed that CG31030 is

167 mainly addressed to synaptic areas in the adult brain (D) and larval CNS (E), as indicated by its apparent

168 colocalization with the presynaptic marker Cadherin-N (CadN). OL: optic lobe, AL: antennal lobe, SO:

169 subœsophageal ganglion. (F) A V5-immunopositive signal was also detected at the neuromuscular

170 junction of $C G 31030^{\mathrm{V} 5}$ larvae, and found to co-localize with anti-horseradish peroxidase (HRP)

171 immunostaining that labels neuronal membranes, confirming the synaptic localization of CG31030. Scale

172 bars: $100 \mu \mathrm{m}$ in $\mathrm{D}$ and $\mathrm{E}$, and $10 \mu \mathrm{m}$ in $\mathrm{F}$.

173

174 CG31030 co-immunoprecipitates with V-ATPase proteins

175 The CG31030 protein is predicted to be part of the InterPro V-ATPase family, but no

176 experimental information is currently available regarding its potential interactors. To determine

177 whether CG31030 could interact, directly or indirectly, with subunits of the V-ATPase complex,

178 we carried out co-immunoprecipitation experiments using an anti-V5 antibody on proteins

179 extracted from heads of $C G 31030^{\sqrt{ } 5}$ mutants and $w^{1118}$ control flies, followed by nano LC-MS/MS

180 mass-spectrometry analysis of the precipitated proteins. Three independent experiments were

181 performed to increase reliability, and in total, 410 proteins were identified in all three

182 experiments. Among those, only 12 proteins had at least a two-fold abundance difference with

183 the control in all three experiments (Figure 2A and Supplementary Table 2), one of them being,

184 as expected, the co-immunoprecipitation target CG31030. Remarkably, three of the 11 other 
185 proteins were identified as subunits of the V-ATPase complex: Vha100-1, VhaAC39-1 and 186 ATP6AP2 (Figure 2B).

187 Vha100-1 and VhaAC39-1 code for subunits $a$ and $d$ of the $V_{0}$ domain, respectively

188 (Vasanthakumar and Rubinstein, 2020). The subunit $a$, coded by five different genes in

189 Drosophila, is the proton port of the pump (Collins and Forgac, 2020). Among the five isoforms,

190 Vha100-1, which co-immunoprecipitated with CG31030 in our experiments, has been shown to

191 be specifically required in neurons and present at the synapse (Hiesenger et al., 2005). Subunit $d$

192 of $\mathrm{V}_{0}$ is coded by two Drosophila genes: VhaAC39-1 and VhaAC39-2, and only the first co-

193 immunoprecipitated with CG31030. According to FlyAtlas, VhaAC39-1 is expressed in many

194 tissues and enriched in the brain, while VhaAC39-2 seems to be mostly found in testis and

195 salivary glands. For both $\mathrm{V}_{0}$ subunits, CG31030 thus co-precipitated with the likely neuronal

196 isoform. The co-immunoprecipitated V-ATPase subunit which appeared to be the most enriched

197 in the $\mathrm{CG} 1030^{\mathrm{V} 5}$ sample was interestingly the accessory subunit ATP6AP2, suggesting a possible

198 direct interaction between this protein and CG31030 (Supplementary Table 2). These

199 experiments therefore reinforce the hypothesis that CG31030 directly interacts with the

200 neuronal V-ATPase complex, and more specifically with $V_{0}$ since all detected partners belong, or

201 interact, with this domain.

202 


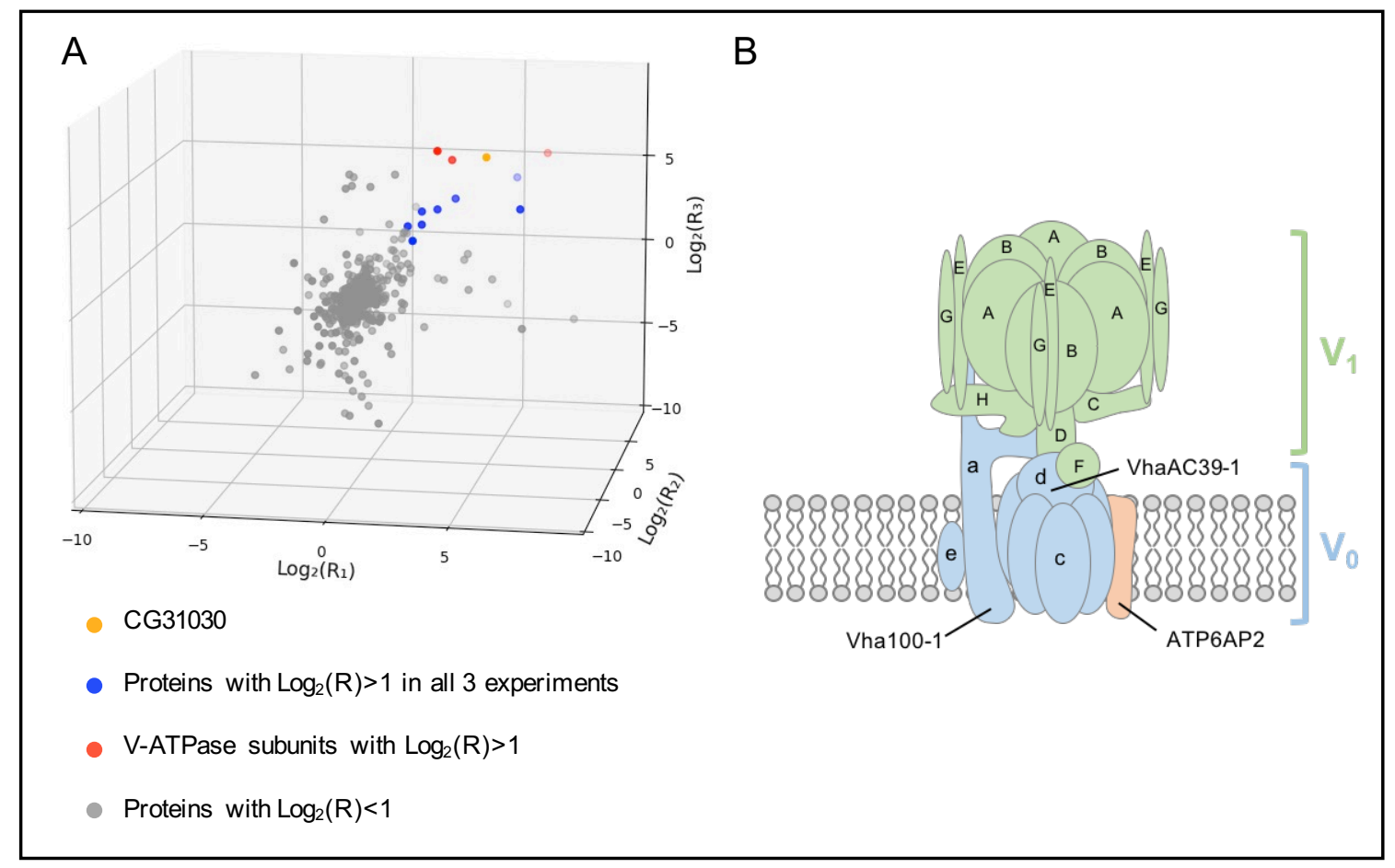

204 Figure 2. CG31030 co-immunoprecipitates with V-ATPase subunits. (A) Scatter plot of proteins identified

205 by nano LC-MS/MS in three independent co-immunoprecipitation experiments using anti-V5 antibodies.

$206 R_{1}$ and $R_{2}$ represent the abundance ratio of proteins identified in adult head extracts from CG31030 ${ }^{\mathrm{v} 5}$

207 over $w^{1118}$ control in experiments 1 and 2 , respectively. Solid lines indicate $\log _{2}(R)=1$, which

208 corresponds to a two-fold abundance difference. 12 proteins were found to be at least twice as

209 abundant in CG31030 ${ }^{\mathrm{v} 5}$ as in the control in all three experiments (red or blue dots on the graph). One of

210 them is the immunoprecipitation target CG31030 (yellow dot) and three of these proteins belong to the

211 V-ATPase complex (red dots). A list of these 12 proteins with their $\log _{2}(R)$ values is provided in

212 Supplementary Table 2. (B) Standard model of the Drosophila V-ATPase complex showing structure of

213 the V1 and V0 domains and the predicted localization of the three subunits that co-immunoprecipitated

214 with CG31030. 


\section{CG31030 knockdown increases the pH of synaptic vesicles}

218 Because CG31030 appeared to be mainly localized in synaptic areas (see Figure 1), we chose to 219 look at the physiological effect of its disruption at the Drosophila larval neuromuscular junction,

220 a model that has contributed to the study of many essential synaptic processes. At synaptic

221 nerve endings, a prominent role of the V-ATPase is to acidify the lumen of synaptic vesicles, the

222 electrochemical gradient generated providing the driving force to load and concentrate the

223 neurotransmitters. Thus, a malfunction of synaptic V-ATPase should induce a decrease of

224 neurotransmitter concentration inside the vesicles, potentially resulting in an altered synaptic

225 transmission. To test this hypothesis, we co-expressed each of the two strongest CG31030 RNAi

226 constructs together with VMAT-pHluorin, a pH-sensitive probe targeted to synaptic vesicles (Wu

227 et al., 2013), in larval motoneurons using the glutamatergic driver OK371-Gal4. Both RNAi1 and

228 RNAi2 induced a lethal phenotype at pupal stage in these conditions. VMAT-pHluorin is an

229 ecliptic pHluorin that is fluorescent at neutral $\mathrm{pH}$, and gets quenched, by protonation, at acidic

$230 \mathrm{pH}$ (Miesenböck et al., 1998). Thus, in control condition, VMAT-pHluorin should not be

231 fluorescent in synaptic vesicles, whose $\mathrm{pH}$ is at around 5.5, but only when externalized on the

232 presynaptic membrane during exocytosis, and so, in contact with the more neutral synaptic cleft

233 milieu. In the case of an acidification defect of synaptic vesicles, the probe could be fluorescent

234 both in synaptic vesicles, where $\mathrm{pH}$ would be abnormally high, and on the presynaptic

235 membrane (Figure 3A, central panel).

236 In order to evaluate the ratio of the internal fluorescence (from synaptic vesicles) over the

237 external fluorescence (from the presynaptic membrane) at the neuromuscular junction of

238 CG31030 knockdown larvae compared to controls, we first quenched the external signal by 
239 replacing the physiological milieu by an identical one with pH adjusted to 5.5 (Figure 3A, left

240 panel). This operation resulted in only the internal signal being conserved. In controls, this

241 meant that all signal was abolished, as expected because the synaptic vesicles were normally

242 acidified (Figure 3B, left panel). In contrast and strikingly, a residual signal was still visible in this

243 acidic milieu in both RNAi1 and RNAi2 knockdown larvae (Figure 3B, right panel). Quantification

244 of the ratio of fluorescence area in acidic milieu over neutral milieu showed that about $37 \%$ of

245 the total signal remained visible in the RNAi larvae after external quenching (Figure 3C and D).

246 To verify that the residual signal seen in knockdown larvae was indeed coming from inside

247 vesicles, the opposite strategy was used: instead of quenching the outside signal, we revealed

248 all the internal one by collapsing the $\mathrm{pH}$ gradient of synaptic vesicles (Figure 3A, right panel). To

249 do so, we replaced the physiological milieu by an ammonium solution, as previously described

250 (Poskanzer and Davis, 2004). This solution had the same composition except that $50 \mathrm{mM} \mathrm{NaCl}$

251 were replaced by $50 \mathrm{mM} \mathrm{NH}_{4} \mathrm{Cl}$. Ammonium and ammonia being in equilibrium $\left(\mathrm{NH}_{4}{ }^{+} \rightleftarrows \mathrm{NH}_{3}+\right.$

$252 \mathrm{H}^{+}$), uncharged ammonia crosses membranes and binds to protons leading to an alkalization of

253 vesicle lumen $\mathrm{pH}$. This increase of vesicular $\mathrm{pH}$ is maintained during $\mathrm{NH}_{4} \mathrm{Cl}$ exposure. In control

254 condition, this gradient collapse should reveal the VMAT-pHluorin probe present in synaptic

255 vesicles and thus highly increase the fluorescent signal. On the contrary, in the case of an

256 acidification defect, the signal should remain fairly stable since synaptic vesicles are already

257 fluorescent. Results were consistent with this hypothesis: while the fluorescence of controls

258 increased about 3 folds in the $\mathrm{pH}$ collapsing ammonium solution, the signal in CG31030

259 knockdown larvae hardly rose by 10 to $20 \%$ depending on the RNAi construct (Figure $3 E, F$ and 


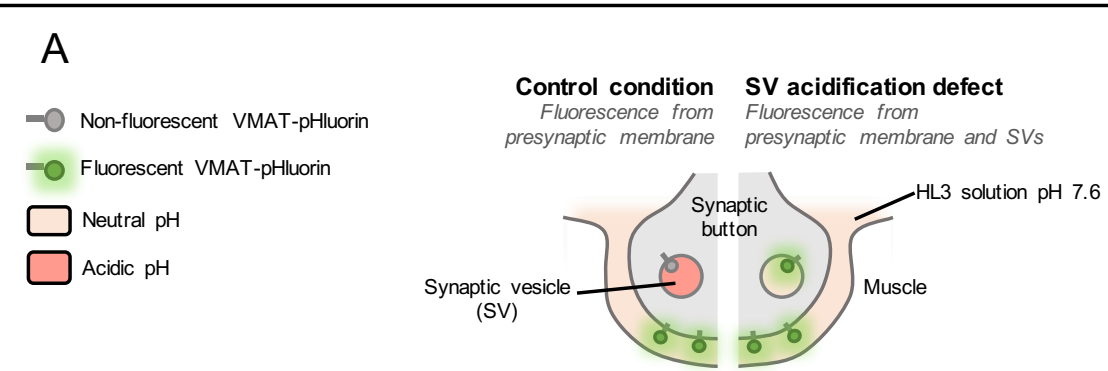

HL3 solution $\mathrm{pH} 5.5$

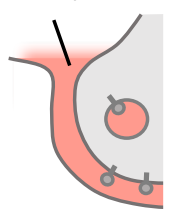

Control condition SV acidification defect

No fluorescence Fluorescence from SVs
$\mathrm{HL} 3-\mathrm{NH}_{4} \mathrm{Cl} \quad \mathrm{pH} 7.6$

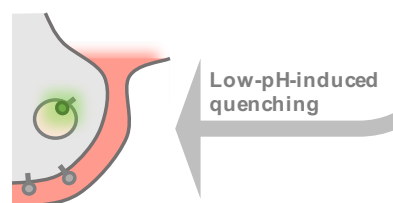

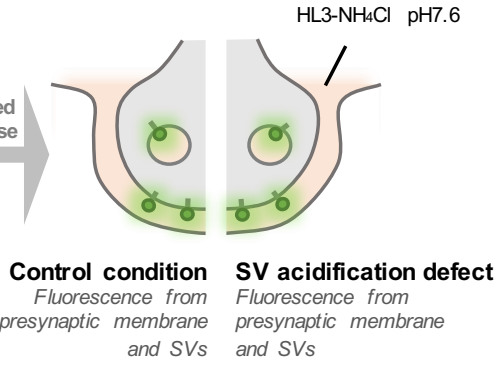

B

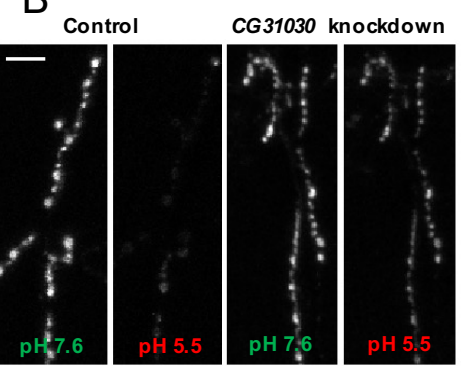

C
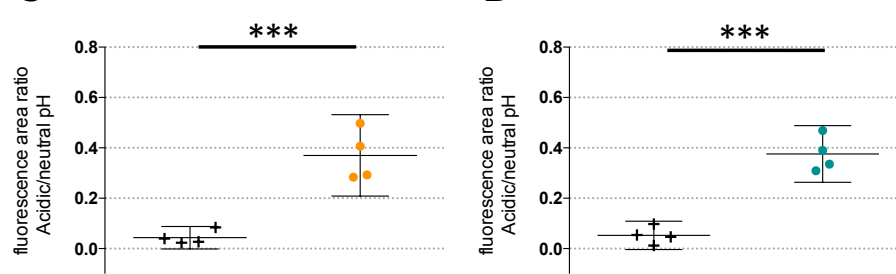

$\mathrm{F}$

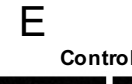

CG31030 knockdown

G
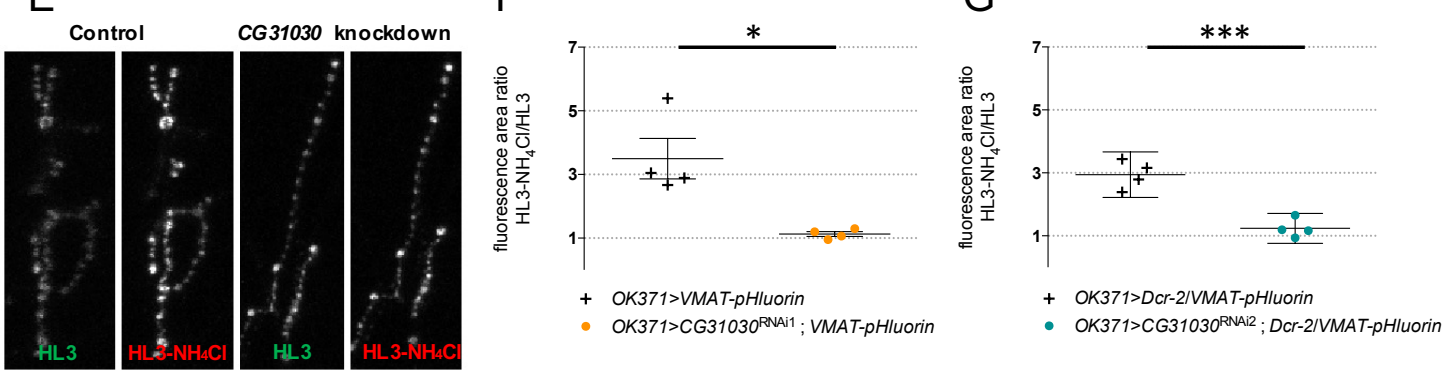

264 Figure 3. CG31030 knockdown larvae have a synaptic vesicle acidification defect. (A) Schematic

265 representation of the protocols used to assess relative acidity levels of synaptic vesicles at the larval neuromuscular junction. (Top center diagram) In control conditions, fluorescence can be emitted by 
267 VMAT-pHluorin in the presynaptic membrane but not in synaptic vesicles since their lumen is acidified. In

268 case of defective synaptic vesicle acidification, both the presynaptic membrane and synaptic vesicles

269 should emit fluorescence. (Left diagram) The fluorescence emitted by VMAT-pHluorin in the presynaptic

270 membrane can be quenched by replacing the extracellular medium with an acidic HL3 solution. This

271 quenching should result in an almost complete extinction of the signal in control flies, in which synaptic

272 vesicles are normally acidified, while a residual signal is expected to be visible in flies having a synaptic

273 vesicle acidification defect. (Right diagram) Replacement of $50 \mathrm{mM} \mathrm{NaCl}$ by $50 \mathrm{mM} \mathrm{NH}_{4} \mathrm{Cl}$ in the neutral

$274 \mathrm{HL} 3$ solution should lead to a collapse of the $\mathrm{pH}$ gradients due to the free diffusion of NH3 in

275 membranes, so that fluorescence will be emitted both by the presynaptic membrane and synaptic

276 vesicles both in control and mutant conditions. (B) Representative pictures showing the effect of

277 perfusing an acidic HL3 solution on VMAT-pHluorin fluorescence in control and CG31030 knockdown

278 larvae. (C, D) Quantification of the ratio of the fluorescence level at pH 5.5 over the original signal at pH

279 7.6. Whereas the low pH extinguished fluorescence in control flies, about $37 \%$ of the signal persisted

280 after quenching in CG31030 knockdown larvae using two different RNAi constructs. (E) Representative

281 pictures showing the effect of collapsing the synaptic vesicle $\mathrm{pH}$ by perfusing $\mathrm{HL} 3-\mathrm{NH}_{4} \mathrm{Cl}$ in control and

282 CG31030 knockdown larvae. $(\mathbf{F}, \mathbf{G})$ Quantification of the ratio of the signal in $\mathrm{HL} 3-\mathrm{NH}_{4} \mathrm{Cl}$ over the original

283 signal in HL3 showed that fluorescence increased about 3-fold in controls while it only rose by 10 to $20 \%$

284 depending on the RNAi in CG31030 knockdown larvae. Results of four independent experiments, with 3-

2855 larvae analyzed per genotype in each experiment. Unpaired Student's $t$-test, ${ }^{*} p<0.05 * * p<0.01$.

286 Mean values with 95\% confidence intervals are reported on the graphs. Scale bar: $15 \mu \mathrm{m}$ in $\mathrm{B}$ and $\mathrm{E}$. 


\section{CG31030 downregulation decreases larval locomotor performance}

291 A reduced $\mathrm{pH}$ gradient of synaptic vesicles in larval motoneurons could alter synaptic

292 transmission and, consequently, the larval locomotor behavior. To assess locomotion, we

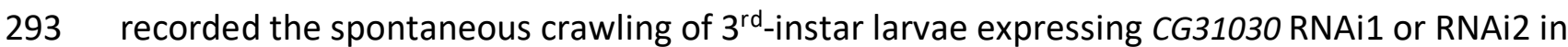

294 motoneurons with OK371-Gal4 on an agar plate with no food source for 2-min periods. Tracking

295 was then performed using the FIMtrack software (Risse et al., 2014), allowing measurement of

296 the total distance travelled and of the stride size, defined as the distance crawled during one

297 peristaltic wave of muscle contraction, and duration (Figure 4A). The knockdown larvae

298 obviously moved less than controls on the plate, as confirmed by their trajectory maps (Figure

$2994 \mathrm{~B}$ and $\mathrm{C}$ ), travelling a distance only half as long as controls with both RNAi (Figure 4E and F).

300 This effect was not simply due to a difference in larval size, as the average length and width of

301 recorded larvae were not significantly different (Supplementary Figure 3).

302 In contrast, the stride size actually showed little or no difference, depending on the RNAi,

303 between knockdown larvae and controls (Figure 4G and H). Instead, we found that the stride

304 duration, which is the time necessary to accomplish one peristaltic wave, was significantly

305 longer in knockdown animals compared to controls for both RNAi (Figure $4 \mathrm{I}$ and J). These results

306 suggest that the knockdown larvae may need about twice as much time as controls to reach the

307 required level of muscular contraction to accomplish one stride. This could be a compensatory

308 mechanism to adapt to a lower amount of neurotransmitter released in response to motor

309 nerve stimulation, as would be expected if synaptic vesicles are less filled with neurotransmitter

310 in CG31030 knockdown context. 
A
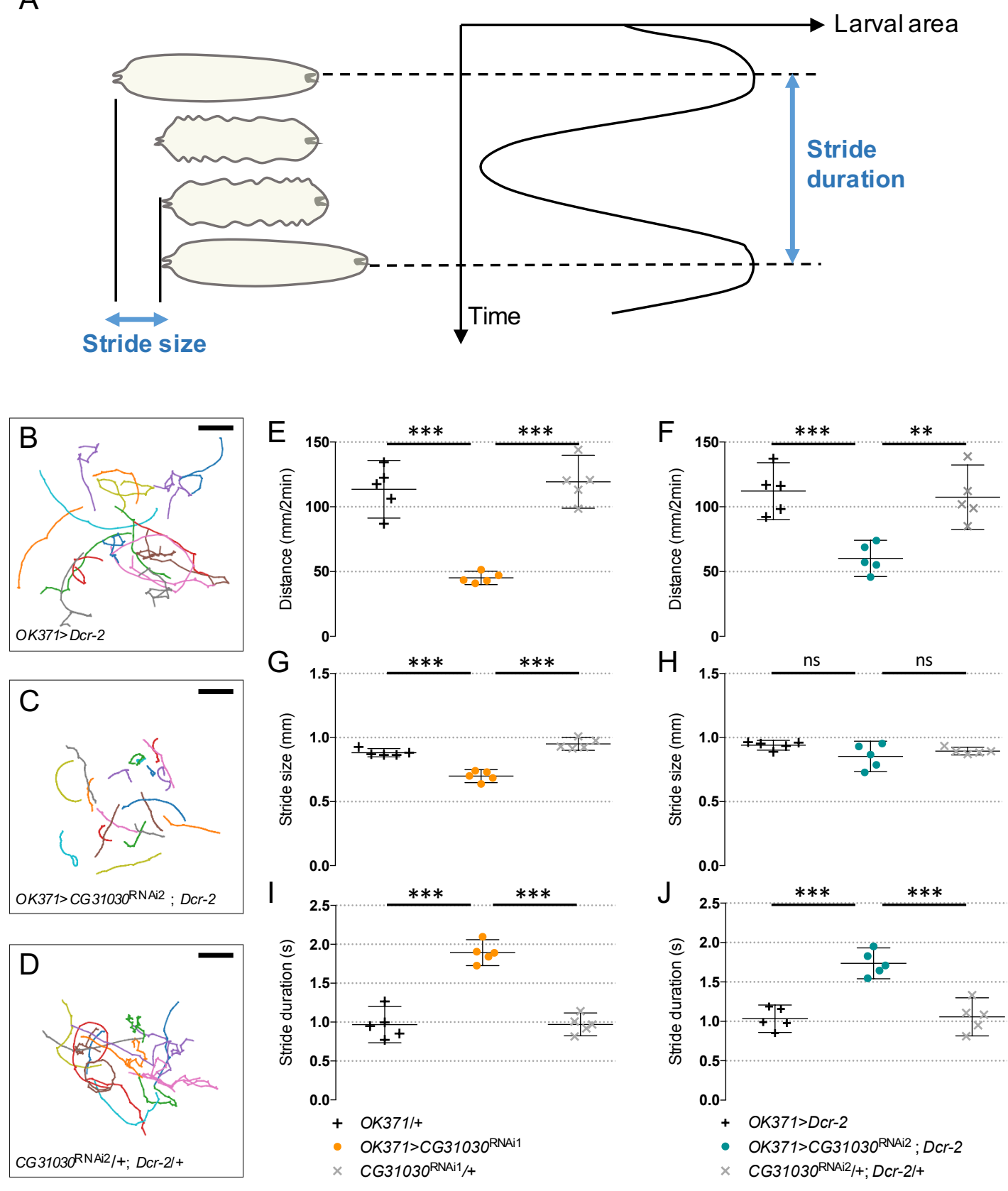

313 Figure 4. CG31030 downregulation in motoneurons decreases larval locomotor performance. (A)

314 Schematic representation of the successive phases of larval locomotion. Stride size is defined as the

315 distance crawled during one peristaltic wave of muscle contraction, while stride duration is the time

316 necessary for the completion of one peristaltic wave. (B-D) Locomotor trails of individual larvae recorded

317 over a period of 2 min. Larvae expressing CG31030 RNAi in motoneurons show reduced spontaneous 
318 movements (C) compared to the driver and UAS controls (B and D, respectively). Scale bar: $25 \mathrm{~mm}$. (E, F)

319 Quantification of the travelled distances confirmed that knocking down CG31030 in motoneurons with

320 two different RNAi induced significant locomotor defects. (G-J) Stride size (G, H) appears to be much less

321 affected than stride duration (I, J) in the knocked-down larvae. Result of five independent experiments,

322 with 3-4 larvae analyzed per genotype in each experiment. One-way ANOVA with Dunnett's post-test for 323 multiple comparisons, ${ }^{* *} p<0.01,{ }^{* * *} p<0,001$. Mean values with $95 \%$ confidence intervals are reported 324 on the graphs.

325

326 Quantal size is reduced in CG31030 knockdown larvae

327 The locomotor deficit of CG31030 knockdown larvae could originate from an impairment of 328 synaptic transmission at the neuromuscular junction or a defect in the central control of motor

329 behavior, or both. To determine if neurotransmission was affected, we carried out

330 electrophysiological recordings to measure the quantal size, which is the postsynaptic response

331 to the release of one synaptic vesicle, at the larval neuromuscular junction. We expressed

332 CG31030 RNAi1 or RNAi2 in motoneurons with the OK371-Gal4 driver, and recorded

333 spontaneous miniature excitatory postsynaptic potentials (mEPSPs) intracellularly from ventral

334 longitudinal abdominal muscle 6 of segment A3 (Figure 5A). These muscles are innervated by

335 synaptic boutons that are clearly marked by OK371-Gal4, as shown by the co-localization of

336 membrane-associated GFP and the postsynaptic marker Discs large (Dlg) in OK371>mCD8::GFP

337 flies (Figure 5B). Representative amplitude distributions of mEPSPs in a control and RNAi1 larvae

338 are shown in Figure 5C. Quantal analysis of recorded events confirmed that both RNAi1 and

339 RNAi2 knockdown larvae have a significantly reduced quantal size compared to controls (Figure

340 5D), suggesting a decrease in glutamate vesicular uptake, and potentially linking vesicle 
341 acidification defect and locomotor impairment. The V-ATPase dysfunction in synapses of

342 CG31030 knockdown larvae apparently led to incomplete loading of vesicles, thus decreasing

343 the amount of neurotransmitter released per unit of time during a peristaltic wave, and

344 potentially slowing down larval locomotion. Although the mean frequency of mEPSPs of both

345 RNAi1 and RNAi2 larvae appeared lower than controls, this effect was not statistically significant

346 (Figure 5E). This result suggests that CG31030 knockdown did not significantly increased the

347 number of unacidified vesicles empty of neurotransmitter. 
A

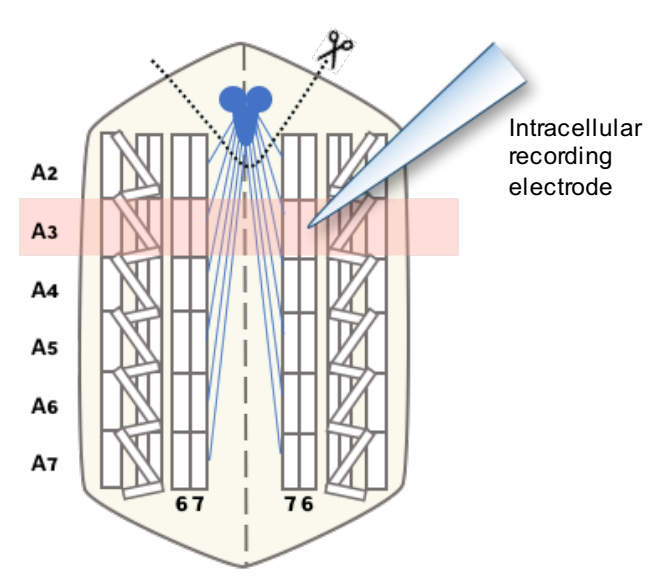

C
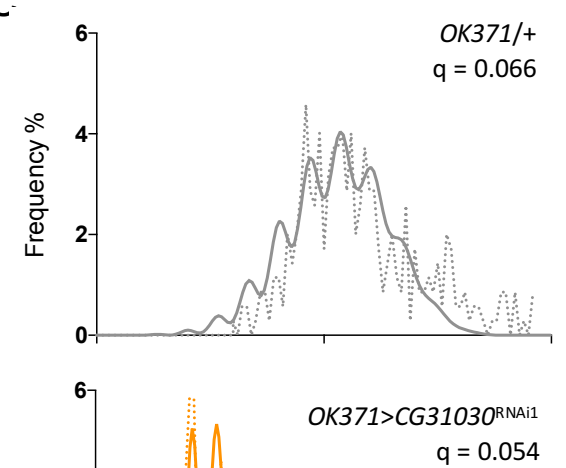

$E$

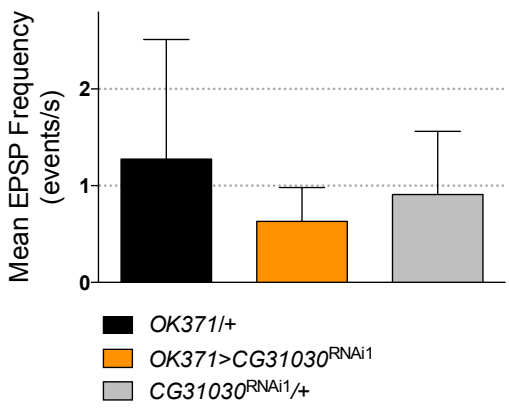

B

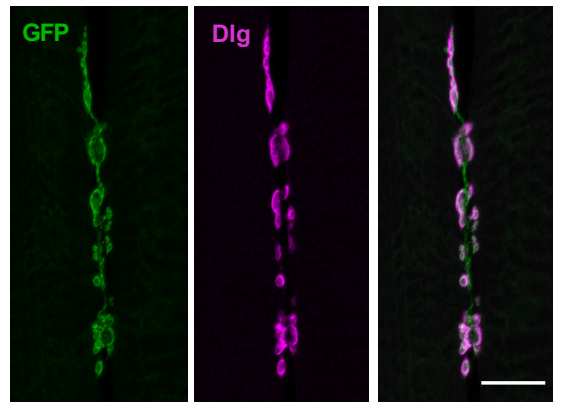

D
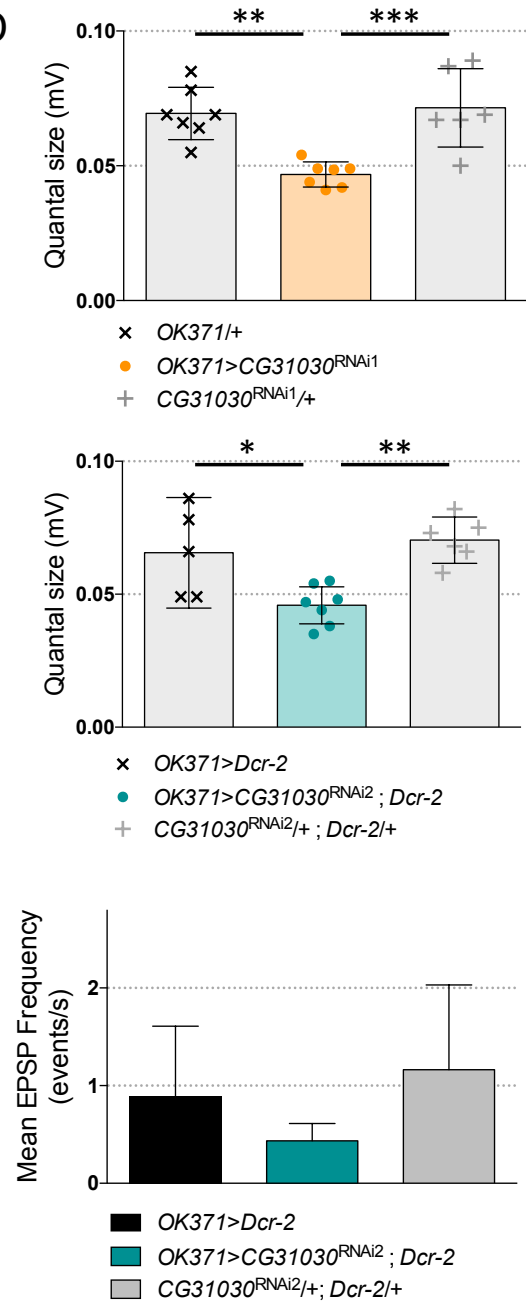

350 Figure 5. Synaptic quanta size is reduced in CG31030 knockdown larvae. (A) Schematic representation of

351 a dissected larval fillet. Spontaneous excitatory postsynaptic potentials (EPSPs) were recorded 
352 intracellularly from the ventral longitudinal abdominal muscle 6 in segment A3. (B) Expression of

353 membrane-associated mCD8::GFP with the glutamatergic driver OK371-Gal4 strongly labels the

354 presynaptic nerve endings at the neuromuscular junction of muscles 6-7 in segment $A 3$. The scaffolding

355 protein Discs large (Dlg) was used as a postsynaptic marker. Scale bar: $20 \mu \mathrm{m}$. (C) Representative

356 distributions of spontaneous mEPSPs recorded in a control larva (top panel, in grey) and a CG31030 RNAi

357 knockdown larva (bottom panel, in orange). The dotted lines represent actually recorded amplitudes and

358 the plain lines the computed theoretical distributions. Genotypes and quantal size (q) are indicated on

359 each graph. (D) Quantal analysis of recorded events showed that knockdown larvae have a significantly

360 reduced synaptic quanta size compared to controls, both with CG31030 RNAi1 (top panel, in orange) or

361 RNAi2 (bottom panel, in blue). (E) Although both RNAi1 (left panel) and RNAi2 (right panel) larvae had a

362 lower mean EPSP frequency than controls, this difference was not statistically significant. One-way

363 ANOVA with Dunnett's post-test for multiple comparisons, ${ }^{*} p<0.05,{ }^{* *} p<0.01,{ }^{* * *} p<0.001$. Mean

364 values with $95 \%$ confidence intervals are reported on the graphs.

367 Discussion

368 In this study, we investigated the hypothesis that the previously uncharacterized Drosophila

369 protein CG31030 is a specific regulator of the neuronal V-ATPase. At variance with its broadly

370 expressed paralog VhaAC45, we have shown that CG31030 is found mainly, if not only, in

371 neurons. We also provide evidence that CG31030 interacts with two constitutive subunits and

372 one accessory subunit of the V-ATPase, the constitutive ones being also enriched in neurons,

373 and that it is required to have properly acidified synaptic vesicles. This implies that CG31030 is

374 an essential protein for nervous system functioning in Drosophila. 


\section{CG31030 is an essential synaptic protein}

377 In yeast, all V-ATPase subunits are coded by a single gene, with the exception of the $V_{0}$ subunit

378 a. The knock-out of any of the single-gene subunits all present a similar phenotype: the inability

379 to survive in a neutral pH environment (Nelson, 2003). For subunit $a$, the same phenotype was

380 only achieved in a double-mutant of both isoforms (Manolson et al., 1994). In multicellular

381 organisms, mutations of V-ATPase subunits, or accessory proteins, also often lead to a lethal

382 phenotype at various developmental stages, whether in mice (Inoue et al., 1999; Sun-Wada et

383 al., 2000; Schoonderwoert et al., 2002-b), C. elegans (Lee et al., 2010), or flies (Davies et al.,

384 1996; Allan et al., 2005). Similarly, a CG31030 null mutant was found to be embryonic lethal,

385 and, interestingly, this lethality could be rescued to the adult stage by re-expressing CG31030 in

386 all neurons, showing that the protein is specifically required in this cell type for fly survival. The

387 percentage of adult survivors was about three times less than what would be expected in case

388 of a full rescue (Supplementary Table 1) and the missing two-thirds likely died at an early

389 developmental stage as no larval lethality was observed. This could be explained by the fact that

390 CG31030 protein re-expression in rescued knock-out flies first required the expression of Gal4

391 under regulation of the elav promoter, which starts to express rather late in embryos, followed

392 by activation of the UAS sequence upstream the CG31030 insert. So, it is possible that part of

393 the mutant embryos did not survive the delay inherent to this ectopic expression process.

394 We found that CG31030 transcripts follows the repartition of the nervous system, being mainly

395 expressed in the head. Moreover, pan-neuronal expression of CG31030 ${ }^{\mathrm{RNAi}}$, the RNAi construct

396 with the weakest effect on fly survival, was sufficient to decrease by more than $80 \%$ its level in 
397 the brain of adult escapers. These results indicate that CG31030 expression is mainly neuronal,

398 if not even entirely restricted to neurons. In addition, CG31030 cellular localization shows

399 similarity with a synaptic pattern. Cell bodies were also marked, although generally less

400 strongly. Synaptic protein complexes can be assembled in the cell bodies before being

401 transported in axons, and it is the case for the soluble and membrane-bound domains of

402 synaptic V-ATPase, as was shown in Torpedo (Morel et al., 1998). It is difficult to decide whether

403 the signal coming from cell bodies corresponds to a functional site for CG31030 or rather to

404 newly produced proteins that have not yet been targeted to synapses. However, the prominent

405 signal in synaptic areas led us to assume that CG31030 plays an important role in the synaptic

406 process.

407

408 CG31030 is required for synaptic vesicle acidification

409 In presynaptic terminals, the most abundant organelles are synaptic vesicles, which require

410 acidification to provide the driving force for neurotransmitter loading. This acidification is

411 ensured by the V-ATPase pump, which generates an electrochemical gradient by importing

412 protons into the vesicle lumen, thus creating both a membrane potential $(\Delta \Psi$, inside positive

413 voltage) and a $\mathrm{pH}$ gradient ( $\Delta \mathrm{pH}$, acidic lumen). Our experiments showed that the knockdown of

414 CG31030 at the glutamatergic larval neuromuscular junction increased the internal pH of

415 synaptic vesicles, and so decreased the $\Delta \mathrm{pH}$ component of the electrochemical gradient

416 generated by the V-ATPase. This result is however by itself insufficient to conclude to a

417 dysfunction of the proton pump, since other players have been shown to influence synaptic

418 vesicle $\mathrm{pH}$ gradient downstream V-ATPase activity. For example, cation $/ \mathrm{H}^{+}$exchangers, found on 
419 synaptic vesicles, can decrease $\Delta \mathrm{pH}$ while increasing $\Delta \Psi$ by exchanging cations, like $\mathrm{Na}^{+}$or $\mathrm{K}^{+}$,

420 against luminal $\mathrm{H}^{+}$(Takamori, 2016). This activity can be upregulated by increased intracellular

421 concentration of these cations (Huang and Trussell, 2014). Glutamate being negatively charged,

422 its transport across vesicular membrane is predominantly driven by $\Delta \Psi$ (Maycox et al., 1988;

423 Tabb at al., 1992). As a consequence, the increased membrane potential resulting from up-

424 regulation of cation/H+ exchangers actually facilitates glutamate uptake (Goh et al., 2011;

425 Huang and Trussell, 2014). We measured synaptic quanta size by quantal analysis of mEPSPs of

426 CG31030 knockdown larvae and found that it was decreased, contrary to what would be

427 expected in the case of an upregulation of cation/ $\mathrm{H}^{+}$exchanger activity. This apparent decrease

428 of vesicular glutamate concentration was corroborated by its phenotypical consequence,

429 namely the locomotion defect exhibited by knockdown larvae. This suggests that the observed

430 diminution of $\Delta \mathrm{pH}$ is correlated with a diminution of $\Delta \Psi$, thus pointing towards a V-ATPase

431 malfunction. This hypothesis is further supported by the co-immunoprecipitation of CG31030

432 with three V-ATPase subunits of the $V_{0}$ domain (Vha100-1, VhaAC39-1 and ATP6AP2), all of

433 them being associated with the neuronal V-ATPase. Altogether, these results strongly suggest

434 that CG31030 is a neuronal protein necessary to V-ATPase function in synapses.

435 Some V-ATPase subunits isoforms in other species have been shown to target specific

436 subcellular compartments, like the Torpedo $\mathrm{V}_{0}$ a1 isoform which is found in synaptic V-ATPase

437 and not in neuronal cell bodies (Morel et al., 2003). Similarly, CG31030 could be specifically

438 involved in the regulation of synaptic V-ATPase, or, alternatively, globally act on all neuronal V-

439 ATPase. Specificity level could even be pushed further, since synaptic vesicles are not the only

440 organelles requiring acidification that are localized in synapses. Thus, a synaptic V-ATPase 
441 regulator could be devoted to synaptic vesicles as it could be affecting more generally all

442 synaptic V-ATPase complexes, indifferently of their membrane localization. The answer to such

443 questions could help better understand regulations of synaptic transmission, since synaptic V-

444 ATPase activity is one of the pre-synaptic modulators of quantal response (Takamori, 2016;

445 Gowrisankaran and Milosevic, 2020).

446

\section{CG31030 interacts with ATP6AP2 and may regulate V-ATPase domain dynamics}

448 While accessory subunits directly interact with V-ATPase domains, other stimulus can indirectly 449 affect activity of the complex, like glucose concentration or serotonin (Sautin et al., 2005; Voss

450 et al., 2007). Co-precipitation of CG31030 with V-ATPase subunits does not necessarily imply a

451 direct interaction, but suggests a minima an indirect association with the complex, possibly in a

452 non-transient manner. The impairment in V-ATPase activity induced by CG31030

453 downregulation rules out the hypothesis of an inhibitory action of this protein on the proton

454 pump. Nevertheless, the precise requirement of CG31030 for activity of the V-ATPase complex

455 still remains unknown to date. Lethality of the mutant could indicate an essential role of

456 CG31030 in V-ATPase function. However, some V-ATPase regulators and accessory subunits

457 have been found in other signaling pathways, like ATP6AP2 in the renin-angiotensin system

458 (Nguyen, 2010), so we cannot exclude that lethality could be due to CG31030 playing a part in

459 other vital neuronal functions. High signal in axons could also point to a role in targeting the

460 complex to the synapses. The two domains of the V-ATPase, V0 and V1, are believed to be

461 assembled in cellular bodies before being separately transported to synaptic area, $V_{0}$ by fast

462 axonal anterograde transport, most likely directly on new synaptic vesicles, and $V_{1}$ by slow 
463 axonal transport like other cytoplasmic synaptic proteins (Morel et al., 1998). CG31030 could be

464 involved in the transport of one of the two domains, and effects of CG31030 knockdown could

465 then result from a decreased synaptic abundance of the affected V-ATPase domain. However, it

466 is believed that only one copy of the V-ATPase complex is sufficient to properly acidify one

467 synaptic vesicle (Takamori et al., 2006), a consequence of this being that neurotransmitter

468 loading would be an all-or-none process. Consistent with this, mutation of the Drosophila

469 synaptic $V_{0}$ subunit Vha100-1 does not seem to impact quantal size but rather mEPSP

470 frequency, potentially reflecting the presence of an increased number of empty synaptic

471 vesicles (Hiesinger et al., 2005). Similarly, a reduction in the frequency of spontaneous quantal

472 events with no change in quantal size was observed when the Drosophila vesicular

473 glutamatergic transporter (VGlut) was downregulated, also suggesting that a single copy of

474 VGlut is sufficient for proper acidification of a vesicle (Daniels et al., 2006). The fact that

475 CG31030 knockdown changes the quantal size but not significantly mEPSP frequency seems to

476 point toward a role of this accessory protein in V-ATPase efficiency, rather than in a process

477 affecting the abundance of the complex such as synaptic targeting.

478 In this respect, it is interesting to note that the V-ATPase protein that more consistently co-

479 immunoprecipitated with CG31030 in our experiments is the accessory subunit ATP6AP2, the fly

480 homolog of human ATP6AP2/PRR. Interestingly, the vertebrate homolog of CG31030,

481 ATP6AP1/Ac45, has also been shown to interact with ATP6AP2, and the complex they form has

482 been proposed to enable the assembly and disassembly of the catalytic and membrane domains

483 of the V-ATPase in the mammalian brain (Rujano et al., 2017; Abbas et al., 2020). This suggests

484 that CG31030 could similarly play a role in the regulation of these dynamic processes, which 
485 may also influence neurotransmitter loading and release in a more continuous way. Our results 486 suggest indeed that modulating V-ATPase activity in presynaptic terminals can finely affect

487 quantal size. Whether such regulation actually occurs in physiological, or pathological,

488 conditions remains to be established. Owing to its structural and functional similarity with its

489 closest Drosophila homolog, VhaAC45/ATP6AP1, we propose therefore to name CG31030

490 "VhaAC45-Like" (VhaAC45L) in further work.

491 Human ATP6AP2/PRR is known to be a Parkinsonism candidate gene and its mutations in

492 humans, mice or flies can lead to cognitive impairment, neurodegeneration and epilepsy

493 (Korvatska et al., 2013; Dubos et al. 2015; Ichihara and Yatabe, 2019). It is therefore of major

494 interest to identify a potential new interactor of ATP6AP2 that is specific to the nervous system,

495 as it could help better understand the consequences of V-ATPase dysregulation on synaptic

496 transmission in pathological contexts. Accordingly, it would be very interesting to determine

497 whether human ATP6AP1 and/or ATP6AP1L share a conserved function with Drosophila

498 CG31030/VhaAC45L in the nervous system. Every new actor identified allows us to paint a more

499 detailed picture of the complex regulations surrounding neuronal V-ATPase specificity,

500 providing new angles for potential therapeutic targets, and a better understanding of

501 fundamental processes such as synaptic transmission.

502

503

504 Materials and methods

505 Drosophila strains, construction and culture

506 Flies were raised on standard agar-cornmeal-yeast medium, at $25^{\circ} \mathrm{C}$ in a $12: 12 \mathrm{~h}$ light-dark cycle. 
507 CG31030 RNAi strains and mutants were obtained from the Bloomington Drosophila stock

508 center (BDSC) and the Vienna Drosophila Resource Center (VDRC). Detailed genotypes and

509 references of these lines are provided in Supplementary Table 3. To construct the UAS-CG31030

510 strain, the CG31030 cDNA was PCR amplified from the clone RH09162 obtained from the

511 Drosophila Genomics Resource Center, Indiana University, USA, using the following primers with

512 added restriction sites: P1-EcoRI (forward) 5'-CCATCCGAATTCAAAATGCAGCTGATTCTCGT and

513 P2-Xhol (reverse) 5'-TGGCTGCTCGAGATCTATTGGGTTATGAGAGA. The $1160 \mathrm{bp}$ PCR fragment

514 was inserted into pUAST (Brand and Perrimon, 1993), verified by sequencing (GATC Biotech) and

515 sent to BestGene, Chino Hills, CA, USA, for P-element transformation by random insertion in

$516 w^{1118}$ background. A 2d-chromosome insertion of UAS-CG31030 that yielded strong expression

517 of the transgene was used thereafter.

519 Reverse transcription-coupled qPCR

520 Total RNAs were extracted from 20 heads (or 15 thoraces or 15 abdomens) of 8-day-old flies

521 using the QIAzol Lysis reagent (Qiagen). The Maxima First Strand cDNA Synthesis Kit (Thermo

522 Fisher Scientific, ref. K1671) was used with oligo(dT)20 primers to synthesize the cDNAs.

523 Relative quantitative PCR assays were carried out using a LightCycler 480 and the SYBR Green I

524 Master mix (Roche LifeScience), with Act5C as internal control for normalization of mRNA levels.

525 All reactions were performed in triplicate. The specificity of amplification products was assessed

526 by melting curve analyses. The following forward and reverse primers, were used: for CG31030,

527 5'-GGCTTCGTTGTAGGCCAACAGA and 5'-CACCAGGTATCCCAAGTTCCAGA; for Act5C, 5'-

528 CGTCGACCATGAAGATCAAG and 5'-TTGGAGATCCACATCTGCTG. 
530 CRISPR/Ca9 gene tagging

531 The sequence of a V5 tag was inserted in frame after the coding sequence of the CG31030 gene,

532 using a homology-directed repair CRISPR-Cas9 method (see Figure 1C). The following guide RNA

533 sequence: 5'-TTCACCGTACAGGAGTAAGG-3' was cloned into the Bbsl site of pCFD3: U6:3-gRNA

534 plasmid (Port et al., 2014) (kind gift of Hervé Tricoire, Université de Paris, Paris, France). This

535 plasmid was then injected, at a concentration of $500 \mathrm{ng} / \mu \mathrm{L}$, with the following single-stranded

536 oligodeoxynucleotide (ssODN) donor repair template: 5'-

537 GTTCGCGCAGCAAACAGTTGACCTTCACCGTACAGGAGTACGCAGGTAAGCCTATCCCTAACCCTCTCCT

538 CGGTCTAGATTCTACGTAAGGAGGTCATAAGTCTCTGATGAACCAATAGATCTCGGGC-3’' synthetized

539 by Integrated DNA Technology, Leuven, Belgium), also at a concentration of $500 \mathrm{ng} / \mu \mathrm{L}$ (the

540 sequence underlined corresponds to the in-frame V5 tag), into nos-cas9 embryos (genotype $y^{1}$,

$541 P($ nos-cas9, $\left.w+), M(3 \times P 3-R F P . a t t P) Z H-2 A, w^{*}\right)$ (Port et al., 2014). An alanine was added before

542 the $V 5$ tag (dashed underline) to prevent the creation of a potential tyrosine phosphorylation

543 site. Embryo injections were performed by BestGene (Chino Hills, CA, USA). Single Fo flies were

544 crossed over the $T M 6 C(S b)$ balancer to establish stable lines. DNA was then extracted from

545 three flies of each of these independent lines, and V5 insertion events were detected by dot

546 blot using a mouse anti-V5 tag monoclonal antibody (Thermo Fisher Scientific, Ref. R960-25).

547 Positive strains were outcrossed in a $w^{1118}$ background and their genomic DNA was sequenced

548 to check for proper in-frame V5 integration in CG31030. One of these equivalent CG31030 ${ }^{\mathrm{V}}$

549 mutant line was selected for further studies.

550 


\section{Immunohistochemistry}

552 Adult brains of 8-day-old females, or $3^{\text {rd }}$-instar larva CNS, were dissected in Drosophila Ringer's

553 solution or hemolymph-like saline solution (HL3) $(70 \mathrm{mM} \mathrm{NaCl}, 5 \mathrm{mM} \mathrm{KCl}, 1.5 \mathrm{mM} \mathrm{CaCl}, 70 \mathrm{mM}$

$554 \mathrm{MgCl}_{2}, 10 \mathrm{mM} \mathrm{NaHCO}_{3}, 115 \mathrm{mM}$ sucrose, $5 \mathrm{mM}$ trehalose, $5 \mathrm{mM}$ HEPES, with pH adjusted to

555 7.6), respectively, and fixed in $4 \%$ paraformaldehyde (Thermo Fischer Scientific) for one hour.

556 After three 20-min washes in PBS plus 0.5\% Triton X-100 (PBT), brains were blocked in PBT $+2 \%$

557 bovine serum albumin for two hours. They were then incubated in primary antibodies diluted in

558 blocking solution for $24 \mathrm{~h}$ at $4^{\circ} \mathrm{C}$. The primary antibodies used were: mouse monoclonal anti-V5

559 (Thermo Fisher Scientific, ref. R960-25, 1:200) and rat anti-CadN (DSHB, ref.DN-Ex \#8, 1:20).

560 Brains were then washed three times, for 20 min each, in PBT before being incubated in

561 secondary antibodies for 2 hours. Secondary antibodies used were: Alexa Fluor 488 anti-mouse

562 and Alexa Fluor 555 anti-rat (Fisher Scientific, ref.A11029 and ref. A21434 respectively), all

563 diluted at 1:1000. After two 20-min washes in PBT followed by two 20-min washes in PBS, brains

564 were mounted in Prolong Gold Antifade Mountant (Thermo Fisher Scientific, ref. P36930).

565 Imaged were acquired on a Nikon A1R confocal microscope.

566 For immunostaining of the larval muscles and neuromuscular junctions, the Alexa Fluor 488

567 Tyramide SuperBoost Kit (Thermo Fisher, ref. B40912) was used to increase the V5 signal that

568 was otherwise faint in this tissue. The working protocol was as recommended by the

569 manufacturer, with anti-V5 diluted to 1:100, then followed by classical immunostaining, as

570 described above, to co-stain for the nerve terminals with an anti-HRP antibody (Jackson

571 ImmunoResearch, ref. 323-005-021, 1:200).

572 


\section{Longevity assay}

574 About 110 virgin females from each genotype were collected on their hatching day, and placed

575 in clean bottles, with no more than 25 flies per bottle. Flies were transferred in new clean

576 bottles, and survivors were counted every two days for 60 days.

\section{Co-immunoprecipitation}

579 About 200 heads from 8 day-old CG31030 ${ }^{\mathrm{V} 5}$ and $w^{1118}$ flies were lysed using glass beads in 500 $580 \mu \mathrm{L}$ of ice-cold lysis buffer: $50 \mathrm{mM}$ Tris-HCl pH 7.4, 2 mM EDTA, $150 \mathrm{mM} \mathrm{NaCl}$, 0.5\% (vol/vol)

581 IGEPAL CA-630 (Sigma-Aldrich, ref. I3021), 10\% (vol/vol) glycerol, 1 mM PMSF protease inhibitor 582 (Sigma-Aldrich, ref. P-7626) and 1X cOmplete Mini Protease Inhibitor Cocktail (Roche, ref. 583 11836153001). Samples were left on ice, with occasional gentle agitation, for 30 min before 584 being centrifuged at $12,000 \mathrm{rpm}(13,000 \mathrm{~g})$ at $4^{\circ} \mathrm{C}$ for $10 \mathrm{~min}$ to remove insoluble material. 400 $585 \mu \mathrm{L}$ of the supernatants were then added to $50 \mu \mathrm{L}$ of Anti-V5-tag mAb-Magnetic Beads (MBL 586 International, Woburn, MA, USA, ref. M167-11), that had been previously washed as 587 recommended by the manufacturer. Samples were incubated with gentle agitation at $4^{\circ} \mathrm{C}$ for 4 588 hours. The supernatants were removed using a magnetic rack, and beads were washed three 589 times with $500 \mu \mathrm{M}$ of ice-cold lysis buffer before being resuspended in $10 \mu \mathrm{L}$ of milliQ water. 590

\section{Mass spectrometry}

592 The MS/MS analysis was performed at the Proteomics facility of the Institut Jacques Monod 593 (Université de Paris, Paris, France). Briefly, proteins were directly digested on the beads by 594 trypsin (Promega, Madison, WI, USA) overnight at $37^{\circ} \mathrm{C}$ in a $25-\mathrm{mM} \mathrm{NH}_{4} \mathrm{HCO}_{3}$ buffer $(0.2 \mu \mathrm{g}$ 
595 trypsin in $20 \mu \mathrm{L}$ ). The resulting peptides were desalted on a ZipTip $\mu$-C18 Pipette Tips (Pierce

596 Biotechnology, Rockford, IL, USA). Eluates were analyzed using either an Orbitrap Fusion or an

597 Orbitrap Q-Exactive Plus, coupled respectively to a Nano-LC Proxeon 1200 or a Nano-LC Proxeon

598 1000, both equipped with an easy spray ion source (Thermo Fisher Scientific, Waltham, MA,

599 USA). Raw data were processed on Proteome Discoverer 2.4 with the mascot node (Mascot

600 version 2.5.1), with a maximum of 2 missed cleavages authorized, against the Swissprot/TrEMBL

601 protein database release 2019_12 for Drosophila melanogaster. The following post-translational

602 modifications were included as variable: Acetyl (Protein N-term), Oxidation (M),

603 Phosphorylation (STY). Peptide identifications were validated with a 1\% FDR (false discovery

604 rate) threshold calculated with the Percolator algorithm. Label-free quantification was done in

605 TOP 3 abundance calculation mode with pairwise ratio-based calculation and t-test (background

606 based) hypothesis test. Only proteins identified in at least one group in two independent

607 experiments were kept in the analysis. Missing values were set to the minimum abundance of

608 the experiment. A more detailed description of the LC-MS/MS procedure is provided in the

609 Supplementary Information.

610

\section{VMAT-pHluorin experiments}

612 Third-instar larvae expressing VMAT-pHluorin with or without CG31030 RNAi in motor neurons

613 using the OK371-Gal4 driver, were dissected to expose the body wall muscles in Ca ${ }^{2+}$-free HL3

614 saline solution ( $70 \mathrm{mM} \mathrm{NaCl}, 5 \mathrm{mM} \mathrm{KCl}, 70 \mathrm{mM} \mathrm{MgCl}_{2}, 10 \mathrm{mM} \mathrm{NaHCO}$, $115 \mathrm{mM}$ sucrose, $5 \mathrm{mM}$

615 trehalose, 5 mM HEPES, pH 7.6). Two other solutions were used: an acidic $\mathrm{Ca}^{2+}$-free HL3 saline

$616(\mathrm{pH} 5.5)$ and a neutral $\mathrm{HL3}-\mathrm{NH}_{4} \mathrm{Cl}$ saline $(\mathrm{pH}$ 7.6), in which $50 \mathrm{mM} \mathrm{NaCl}$ was replaced by $50 \mathrm{mM}$ 
$617 \mathrm{NH}_{4} \mathrm{Cl}$. After dissection, larvae fillets were allowed to settle in $\mathrm{Ca}^{2+}$-free $\mathrm{HL} 3$ saline for $10 \mathrm{~min}$

618 before being scanned a first time directly in a drop of the solution using a Nikon A1R confocal

619 microscope. $\mathrm{Ca}^{2+}$-free HL3 saline was then replaced either by the acidic $\mathrm{Ca}^{2+}{ }^{+}$free HL3 saline for

620 low $\mathrm{pH}$-induced quenching experiments, or by the $\mathrm{HL} 3-\mathrm{NH}_{4} \mathrm{Cl}$ saline for $\mathrm{pH}$ gradient collapse

621 experiments. In both cases, larval fillets were rinsed three times with the modified solution, and

622 incubated for $3 \mathrm{~min}$, before being scanned a second time, in a drop of modified solution.

623 Quantification was done on Z-projections (set to maximal intensity) of confocal stacks. Using the

624 Fiji software, a fixed threshold was applied to all images to get rid of the background and select

625 only synaptic areas. Percentage of area over the threshold was used as a measure of the signal

626 intensity and ratios of the values obtained in modified solutions over standard HL3, i.e. the

627 second scan over the first scan, were calculated for quantifications.

\section{Larval locomotion assays}

630 Larval locomotion was assessed on an in-house made version of the FIMtable system (Risse et

631 al., 2014). Third-instar larvae were collected and briefly rinsed in water to remove traces of

632 food, before being gently placed on the recording table precoated with a thin layer of $1.2 \%$ agar

633 gel. Only four larvae of the same genotype were recorded simultaneously, to avoid collisions

634 between animals, for a period of 2 min with a Basler ace acA2040-25gm camera at 12.5

635 frames/s. Larvae that burrowed themselves into the agar plate or escaped the arena before the

636 end of recording were excluded from the results. Tracking was done using the FIMtrack

637 software, as described in (Risse et al., 2014). The number of peristaltic waves was computed

638 from the variations in larval area. More precisely, the curves of area variation were first 
639 smoothed with a Savitzky-Golay filter to get rid of unwanted noise, then the number of waves

640 was defined as the number of peaks on the curve (which was automatically computed by a

641 custom-made Python script). Stride size was then calculated as the distance travelled by the

642 larva divided by the number of peristaltic waves, while stride duration was defined as the

643 recording time (i.e. 120 seconds) divided by the number of peristaltic waves.

644

\section{Electrophysiological recording and quantal analysis}

646 Third-instar larvae expressing CG31030 RNAi in motor neurons using the OK371-Gal4 driver, and

647 appropriate controls, were dissected to expose the body wall muscles, and the brain removed,

648 in $\mathrm{Ca}^{2+}$-free $\mathrm{HL} 3$ saline solution $\left(70 \mathrm{mM} \mathrm{NaCl}, 5 \mathrm{mM} \mathrm{KCl}, 70 \mathrm{mM} \mathrm{MgCl}, 10 \mathrm{mM} \mathrm{NaHCO}_{3}, 115 \mathrm{mM}\right.$

649 sucrose, 5 mM trehalose, 5 mM HEPES, pH 7.6) (Stewart et al., 1994; Cattaert and Birman,

650 2001). Spontaneous miniature EPSPs (mEPSPs) were recorded in the presence of tetrodotoxin

651 (TTX) $10^{-6} \mathrm{M}$, so that no spike could occur, from the ventral longitudinal abdominal muscle 6 in

652 segment A3. Quantal analysis was performed following the theoretical background described in

653 (Kuno, 1971) and (Castellucci and Kandel, 1974). Distribution histograms of mEPSP size were

654 built from each muscle fiber recording with a $0.01 \mathrm{mV}$ bin size. These histograms provided an

655 estimate of the mean size of a unitary EPSP, since the peaks represent integer multiples of the

656 unitary size. A theoretical distribution was then computed by convolving a binomial distribution,

657 accounting for quantal content (number of quanta released), and a Gaussian distribution,

658 allowing for variations in size of individual quanta. A detailed description of the mathematical

659 treatment is provided in the Supplementary Information.

660 


\section{1}

662 Statistical analysis was performed with the GraphPad Prism 6 software. The paired Student's t-

663 test was used for comparison of two genotypes, while either paired or unpaired ANOVA, with

664 Dunnett's post-test for multiple comparisons, were used for three genotypes.

\section{Acknowledgements}

668 We thank Dr Philippe Marin for helpful advice about the quantitative analysis of mass

669 spectrometry experiments, Drs Hervé Tricoire and Elodie Martin for helpful suggestion on the 670 CRISPR/Cas9 mutagenesis, Pr David Krantz for providing the UAS-VMAT-pHluorin strain, Dr Hervé 671 Tricoire for the pCFD3: U6:3-gRNA plasmid, and Dr. Céline Petitgas and Manon Dobrigna for 672 participating in preliminary experiments during their student internships. This work was 673 supported by a grant from the Fondation de France (Subvention $n^{\circ}$ 0086407) to SB and recurrent 674 fundings to his lab from the ESPCI Paris and CNRS. AD, ARI and JS were recipient of PhD fellowships 675 from PSL University and Labex MemoLife, the Doctoral School 3C and the China Scholarship 676 Council, respectively.

677

\section{Competing interests}

679 No competing interest to declare.

680

681 


\section{References}

683

Abbas, Y. M., Wu, D., Bueler, S. A., Robinson, C. V., \& Rubinstein, J. L. (2020). Structure of

V-ATPase from the mammalian brain. Science, 367(6483), 1240-1246.

686

Allan, A. K., Du, J., Davies, S. A., \& Dow, J. A. (2005). Genome-wide survey of V-ATPase

genes in Drosophila reveals a conserved renal phenotype for lethal alleles. Physiological

genomics, 22(2), 128-138.

690

Cannata Serio, M., Rujano, M. A., \& Simons, M. (2018). Mutations in ATP6AP2 cause

692 autophagic liver disease in humans. Autophagy, 14(6), 1088-1089.

693

Castellucci, V. F., \& Kandel, E. R. (1974). A quantal analysis of the synaptic depression

695 underlying habituation of the gill-withdrawal reflex in Aplysia. Proceedings of the National

696 Academy of Sciences, 71(12), 5004-5008.

697

698

Cattaert, D., \& Birman, S. (2001). Blockade of the central generator of locomotor rhythm

699 by noncompetitive NMDA receptor antagonists in Drosophila larvae. Journal of neurobiology,

$700 \quad 48(1), 58-73$.

701

702

Chintapalli, V. R., Wang, J., \& Dow, J. A. (2007). Using FlyAtlas to identify better

703 Drosophila melanogaster models of human disease. Nature genetics, 39(6), 715-720. 
$707 \quad 75-88$.

708

709

Collins, M. P., \& Forgac, M. (2020). Regulation and function of V-ATPases in physiology

710 and disease. Biochimica et Biophysica Acta (BBA)-Biomembranes, 183341.

711

712

Daniels, R. W., Collins, C. A., Chen, K., Gelfand, M. V., Featherstone, D. E., \& DiAntonio, A.

713 (2006). A single vesicular glutamate transporter is sufficient to fill a synaptic vesicle. Neuron,

714 49(1), 11-16.

715

716 Davie, K., Janssens, J., Koldere, D., De Waegeneer, M., Pech, U., Kreft, Ł., ... \&

717 Poovathingal, S. (2018). A single-cell transcriptome atlas of the aging Drosophila brain. Cell,

718 174(4), 982-998.

719

720 Davies, S. A., Goodwin, S. F., Kelly, D. C., Wang, Z., Sözen, M. A., Kaiser, K., \& Dow, J. A.

721 (1996). Analysis and inactivation of vha55, the gene encoding the vacuolar ATPase B-subunit in

722 Drosophila melanogaster reveals a larval lethal phenotype. Journal of Biological Chemistry,

$723271(48), 30677-30684$.

724 
726 Journal of physiology, 124(3), 560.

727

Dietzl, G., Chen, D., Schnorrer, F., Su, K. C., Barinova, Y., Fellner, M., ... \& Couto, A.

729 (2007). A genome-wide transgenic RNAi library for conditional gene inactivation in Drosophila.

$730 \quad$ Nature, 448(7150), 151-156.

731

732

Dubos, A., Castells-Nobau, A., Meziane, H., Oortveld, M. A., Houbaert, X., lacono, G., ... \&

733 Bhukel, A. (2015). Conditional depletion of intellectual disability and Parkinsonism candidate

734 gene ATP6AP2 in fly and mouse induces cognitive impairment and neurodegeneration. Human

735 molecular genetics, 24(23), 6736-6755.

736

737 Forgac, M. (2007). Vacuolar ATPases: rotary proton pumps in physiology and

738 pathophysiology. Nature reviews Molecular cell biology, 8(11), 917-929.

739

740 Goh, G. Y., Huang, H., Ullman, J., Borre, L., Hnasko, T. S., Trussell, L. O., \& Edwards, R. H.

741 (2011). Presynaptic regulation of quantal size: $\mathrm{K}+\mathrm{H}+$ exchange stimulates vesicular glutamate

742 transport. Nature neuroscience, 14(10), 1285-1292.

743

744 Gowrisankaran, S., \& Milosevic, I. (2020). Regulation of synaptic vesicle acidification at

745 the neuronal synapse. IUBMB life, 72(4), 568-576.

746 
Guida, M. C., Hermle, T., Graham, L. A., Hauser, V., Ryan, M., Stevens, T. H., \& Simons, M.

748 (2018). ATP6AP2 functions as a V-ATPase assembly factor in the endoplasmic reticulum.

749 Molecular biology of the cell, 29(18), 2156-2164.

750

751

Hiesinger, P. R., Fayyazuddin, A., Mehta, S. Q., Rosenmund, T., Schulze, K. L., Zhai, R. G.,

752 ... \& Bellen, H. J. (2005). The v-ATPase V0 subunit a1 is required for a late step in synaptic vesicle

753 exocytosis in Drosophila. Cell, 121(4), 607-620.

754

755

Huang, H., \& Trussell, L. O. (2014). Presynaptic HCN channels regulate vesicular

756 glutamate transport. Neuron, 84(2), 340-346.

757

758

Ichihara, A., \& Yatabe, M. S. (2019). The (pro) renin receptor in health and disease.

759 Nature Reviews Nephrology, 1.

760

761

Inoue, H., Noumi, T., Nagata, M., Murakami, H., \& Kanazawa, H. (1999). Targeted

762

disruption of the gene encoding the proteolipid subunit of mouse vacuolar H+-ATPase leads to

763 early embryonic lethality. Biochimica et Biophysica Acta (BBA)-Bioenergetics, 1413(3), 130-138.

764

765

Jan, L. Y. \& Jan, Y. N. (1982). Antibodies to horseradish peroxidase as specific neuronal

766 markers in Drosophila and in grasshopper embryos. Proceedings of the National Academy of

767 Sciences USA, 79(8), 2700-2704

768 
Jansen, E. J. R., \& Martens, G. J. M. (2012). Novel insights into V-ATPase functioning:

770 distinct roles for its accessory subunits ATP6AP1/Ac45 and ATP6AP2/(pro) renin receptor.

771 Current Protein and Peptide Science, 13(2), 124-133.

772

773

Jansen, E. J., Timal, S., Ryan, M., Ashikov, A., Van Scherpenzeel, M., Graham, L. A., ... \&

774 Steenbergen, G. (2016). ATP6AP1 deficiency causes an immunodeficiency with hepatopathy,

775 cognitive impairment and abnormal protein glycosylation. Nature communications, 7(1), 1-13.

776

777

Korvatska, O., Strand, N. S., Berndt, J. D., Strovas, T., Chen, D. H., Leverenz, J. B., ... \&

778 Bonkowski, E. (2013). Altered splicing of ATP6AP2 causes X-linked parkinsonism with spasticity

779 (XPDS). Human molecular genetics, 22(16), 3259-3268.

780

781

Kuno, M. (1971). Quantum aspects of central and ganglionic synaptic transmission in

782 vertebrates. Physiological Reviews, 51(4), 647-678.

783

784

Lee, S. K., Li, W., Ryu, S. E., Rhim, T., \& Ahnn, J. (2010). Vacuolar (H+)-ATPases in

785 Caenorhabditis elegans: what can we learn about giant $\mathrm{H}+$ pumps from tiny worms?. Biochimica

786 et Biophysica Acta (BBA)-Bioenergetics, 1797(10), 1687-1695.

787

788

Lin, D. M., \& Goodman, C. S. (1994). Ectopic and increased expression of Fasciclin II alters

789 motoneuron growth cone guidance. Neuron, 13(3), 507-523.

790 
792 glutamate transporter: a marker protein for motoneurons and glutamatergic centers in the 793 brain. Gene Expression Patterns, 6(3), 299-309.

794

Manolson, M. F., Wu, B., Proteau, D., Taillon, B. E., Roberts, B. T., Hoyt, M. A., \& Jones, E.

796 W. (1994). STV1 gene encodes functional homologue of 95-kDa yeast vacuolar H (+)-ATPase

797 subunit Vph1p. Journal of Biological Chemistry, 269(19), 14064-14074.

798

799

Maycox, P. R., Deckwerth, T., Hell, J. W., \& Jahn, R. (1988). Glutamate uptake by brain

800 synaptic vesicles. Energy dependence of transport and functional reconstitution in

801 proteoliposomes. Journal of Biological Chemistry, 263(30), 15423-15428.

802

803

Miesenböck, G., De Angelis, D. A., \& Rothman, J. E. (1998). Visualizing secretion and

804 synaptic transmission with pH-sensitive green fluorescent proteins. Nature, 394(6689), 192-195.

805

806

Mitchell, A. L., Attwood, T. K., Babbitt, P. C., Blum, M., Bork, P., Bridge, A., ... \& Gough, J.

807 (2019). InterPro in 2019: improving coverage, classification and access to protein sequence

808 annotations. Nucleic acids research, 47(D1), D351-D360.

809

810 Morel, N., Gérard, V., \& Shiff, G. (1998). Vacuolar H+-ATPase domains are transported

811 separately in axons and assemble in Torpedo nerve endings. Journal of neurochemistry, 71(4), $812 \quad 1702-1708$. 
814 Morel, N., Dedieu, J. C., \& Philippe, J. M. (2003). Specific sorting of the a1 isoform of the $815 \mathrm{~V}-\mathrm{H}+\mathrm{ATPase}$ a subunit to nerve terminals where it associates with both synaptic vesicles and 816 the presynaptic plasma membrane. Journal of Cell Science, 116(23), 4751-4762.

819 endocrine systems. Journal of Experimental Biology, 172(1), 171-178.

820

822 M. C., ... \& Booth, B. W. (2015). A library of MiMICs allows tagging of genes and reversible, 823 spatial and temporal knockdown of proteins in Drosophila. Elife, 4, e05338.

826 ATPase). Journal of bioenergetics and biomembranes, 35(4), 281-289. 
Perkins, L. A., Holderbaum, L., Tao, R., Hu, Y., Sopko, R., McCall, K., ... \& Miller, A. (2015).

836 The transgenic RNAi project at Harvard Medical School: resources and validation. Genetics, $837201(3), 843-852$.

838

839

Port, F., Chen, H. M., Lee, T., \& Bullock, S. L. (2014). Optimized CRISPR/Cas tools for

840 efficient germline and somatic genome engineering in Drosophila. Proceedings of the National

841 Academy of Sciences, 111(29), E2967-E2976.

842

843

Poskanzer, K. E., \& Davis, G. W. (2004). Mobilization and fusion of a non-recycling pool of

844 synaptic vesicles under conditions of endocytic blockade. Neuropharmacology, 47(5), 714-723.

845

846

Risse, B., Otto, N., Berh, D., Jiang, X., \& Klämbt, C. (2014). FIM imaging and FIMtrack: two

847 new tools allowing high-throughput and cost effective locomotion analysis. JoVE (Journal of

$848 \quad$ Visualized Experiments), (94), e52207.

849

850

Rujano, M. A., Cannata Serio, M., Panasyuk, G., Péanne, R., Reunert, J., Rymen, D., ... \&

851 Simons, M. (2017). Mutations in the X-linked ATP6AP2 cause a glycosylation disorder with

852 autophagic defects. Journal of Experimental Medicine, 214(12), 3707- 3729.

853

854

Saroussi, S., \& Nelson, N. (2009). The little we know on the structure and machinery of V-

855 ATPase. Journal of Experimental Biology, 212(11), 1604-1610.

856 
Sautin, Y. Y., Lu, M., Gaugler, A., Zhang, L., \& Gluck, S. L. (2005). Phosphatidylinositol 3-

858 kinase-mediated effects of glucose on vacuolar H+-ATPase assembly, translocation, and

859 acidification of intracellular compartments in renal epithelial cells. Molecular and cellular

860 biology, 25(2), 575-589.

861

862

Schoonderwoert, V. T. G., \& Martens, G. J. (2002-a). Structural gene organization and

863 evolutionary aspects of the V-ATPase accessory subunit Ac45. Biochimica et Biophysica Acta

864 (BBA)-Gene Structure and Expression, 1574(3), 245-254.

865

866

Schoonderwoert, V. T. G., \& Martens, G. J. (2002-b). Targeted disruption of the mouse

867 gene encoding the V-ATPase accessory subunit Ac45. Molecular membrane biology, 19(1), 67-

86871.

869

870

Shao, E., \& Forgac, M. (2004). Involvement of the nonhomologous region of subunit A of

871 the yeast V-ATPase in coupling and in vivo dissociation. Journal of Biological Chemistry, 279(47),

$872 \quad 48663-48670$.

873

874 Stewart, B. A., Atwood, H. L., Renger, J. J., Wang, J., \& Wu, C. F. (1994). Improved stability

875 of Drosophila larval neuromuscular preparations in haemolymph-like physiological solutions.

876 Journal of Comparative Physiology A, 175(2), 179-191.

877 
Tabb, J. S., Kish, P. E., Van Dyke, R., \& Ueda, T. (1992). Glutamate transport into synaptic

879 vesicles. Roles of membrane potential, pH gradient, and intravesicular pH. Journal of Biological

880 Chemistry, 267(22), 15412-15418.

881

882

Takamori, S. (2016). Presynaptic molecular determinants of quantal size. Frontiers in

883

synaptic neuroscience, $8,2$.

884

885

Thurmond, J., Goodman, J. L., Strelets, V. B., Attrill, H., Gramates, L. S., Marygold, S. J., ...

886 \& Kaufman, T. C. (2019). FlyBase 2.0: the next generation. Nucleic acids research, 47(D1), D759-

887 D765.

888

889

Vasanthakumar, T., \& Rubinstein, J. L. (2020). Structure and Roles of V-type ATPases.

890 Trends in Biochemical Sciences, 45(4), 295-307.

891

892

Voss, M., Vitavska, O., Walz, B., Wieczorek, H., \& Baumann, O. (2007). Stimulus-induced

893 phosphorylation of vacuolar H+-ATPase by protein kinase A. Journal of Biological Chemistry,

$894282(46), 33735-33742$.

895

896

Wu, T. H., Lu, Y. N., Chuang, C. L., Wu, C. L., Chiang, A. S., Krantz, D. E., \& Chang, H. Y.

897 (2013). Loss of vesicular dopamine release precedes tauopathy in degenerative dopaminergic

898 neurons in a Drosophila model expressing human tau. Acta neuropathologica, 125(5), 711-725.

899 
901

902 Amina Dulac, Abdul-Raouf Issa, Jun Sun, Giorgio Matassi, Baya Chérif-Zahar, Daniel

903

Cattaert and Serge Birman

904

905

\section{Supplementary information}

906

907 Supplementary figures

908 Supplementary tables

909 Supplementary methods

910

911 


\section{Supplementary figures}

913

A

CG31030 1 M-QLILVATLICLIIGSCSASISGPFIF-WGHSRVSSQQTQALV-ESSSRELPLTQLFTE 57

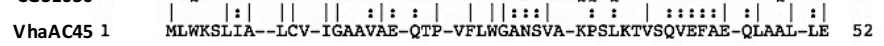

58 AKAI-VVFVRNT-TNRLEGTRYPKFQNLVKSGAWTYLP-QRSLAAAE-P-FGLNANIEVVS 112

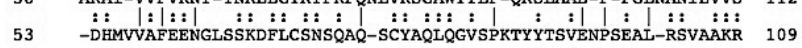

113 LSGHGEEDDSEILLAYNEAVNTYGRGEVLGILGSREEEAHFLAKREAAPGGEEEEKAKAA 172

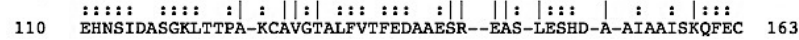

173 EGSEETEASFIYVAEGNKAVLSLNGPLELLRVTNDTLKLEEHIKQITFDDQRAKGYGRLSI 232

164 KVAYYYLA-APSTAPVVQRRTRRDTAATTGGIMWKSTNQFOI-FYTALLYNGNPITVTDL 221

233 TFMH-SGEKCTLRFKFSLIRGSWTLRN-V-EVEYREL-KSVLVARGDEYTLPSAPLGF-S 287

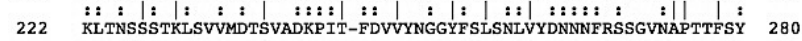

288 YR--CSAESVNFLNPARN-ETIQSLLLSSDFQVQPWLNGRPEYGEVYDCIGFVSAPILAGL 344

281 SCGNLTLESAA-VNNMYNTLSFKSLLLAPFDGTYKEDFP-FGDSWDVGFVTPGILMGL 338

345 FVVTLLLGILGLGISAMLSMHTPNRFESSRSKQLTFTVQE- 384

339 FVVALLVIMFVGVCWMMDINTMDRFDDPKGKTITINAAAE 379

$\mathrm{B}$

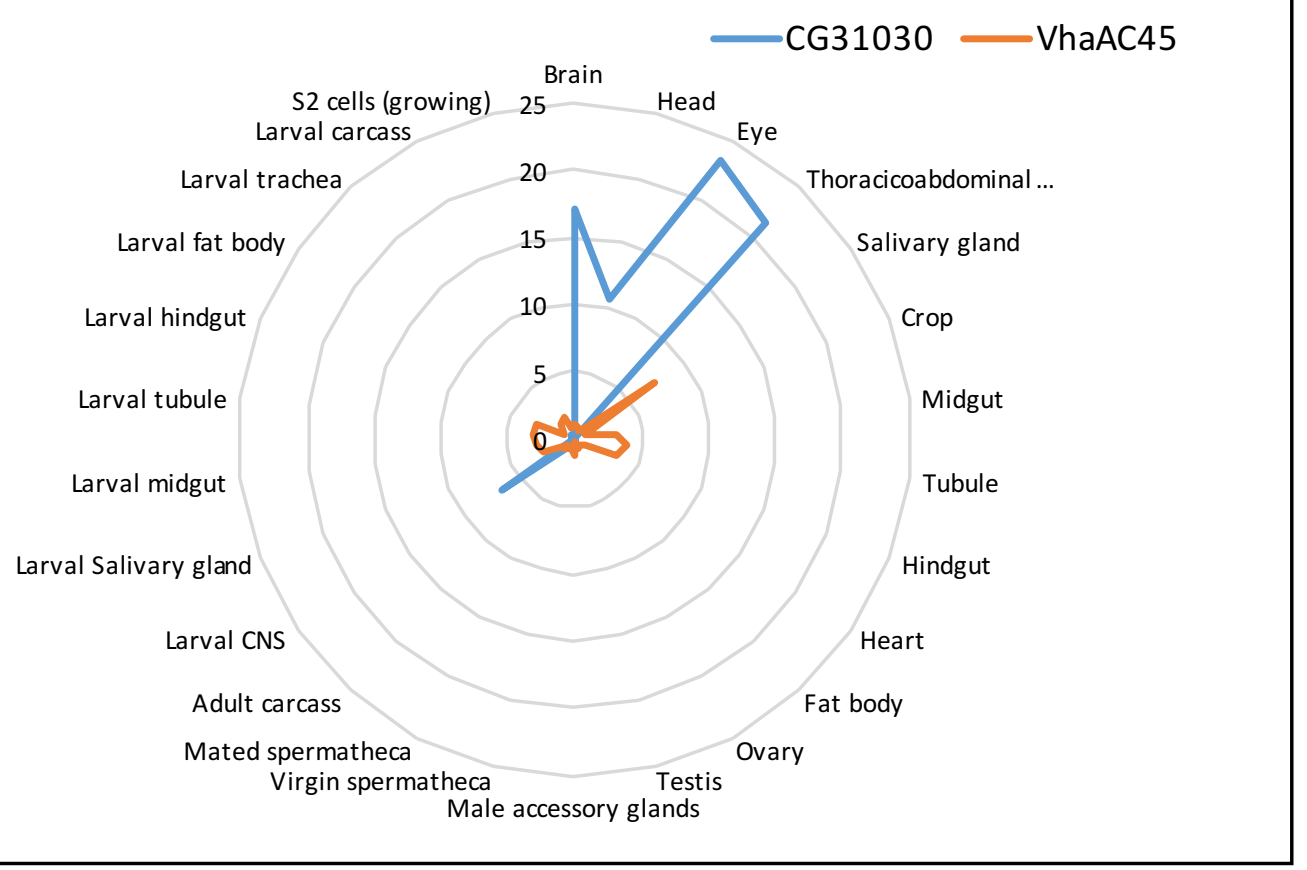

915 Supplementary Figure 1. CG31030 is a paralog of VhaAC45 expressed in the nervous system. (A) Amino-

916 acid sequence alignment shows that CG31030 and VhaAC45 share $69.9 \%$ similarity. (B) Diagram

917 representing expression levels of CG31030 and VhaAC45 in different tissues relative to the whole fly, 
918 according to FlyAtlas data (Chintapalli et al., 2007). CG31030 appears to be markedly enriched in the

919 nervous system of larva and adult fly, whereas in contrast VhaAC45 seems to be uniformly expressed in

920 all tissues in these two stages.

921

922

923

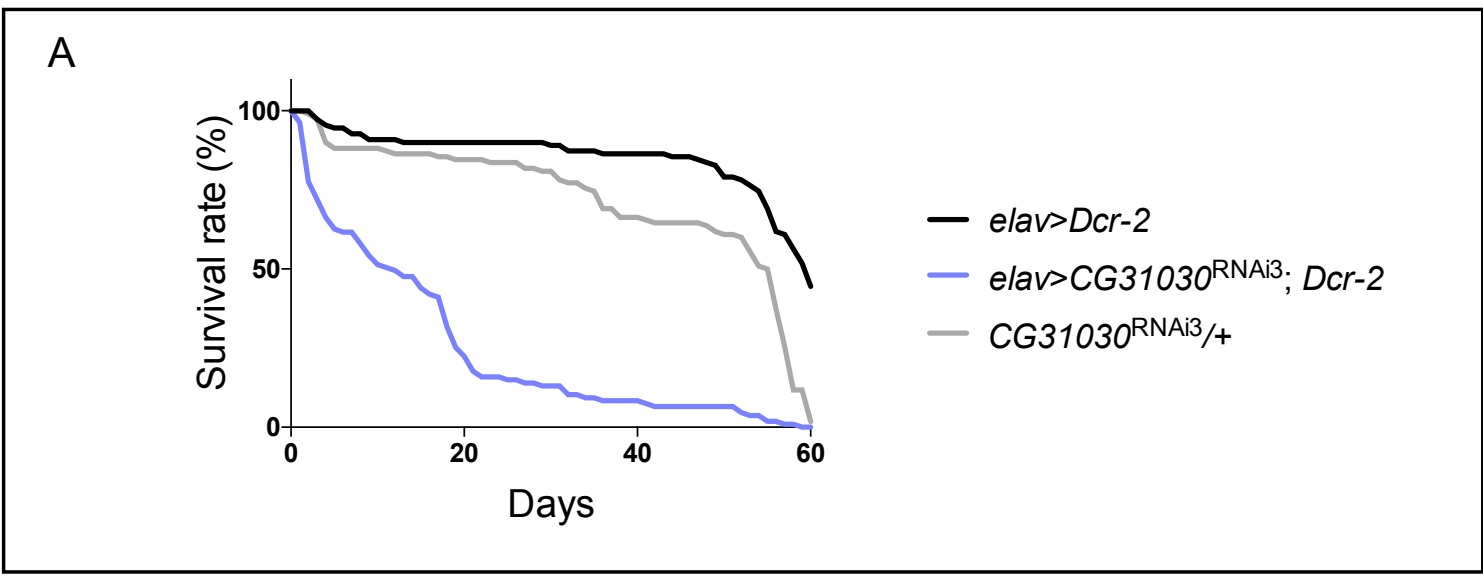

924 Supplementary Figure 2. Knockdown of CG31030 expression in neurons decreases adult longevity. Pan-

925 neuronal expression of $C G 31030^{\mathrm{RNAi3}}$ and Dcr-2 with the elav-Gal4 driver led to a marked shortening of

926 the lifespan of adult flies. Results of one experiment, carried out with 105-110 females per genotype.

927

928

929

930

931

932

933 
A

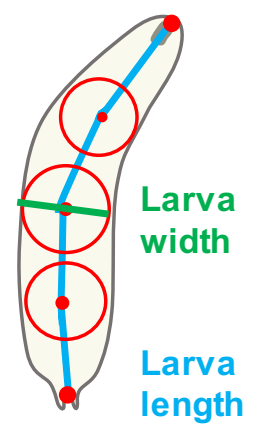

B

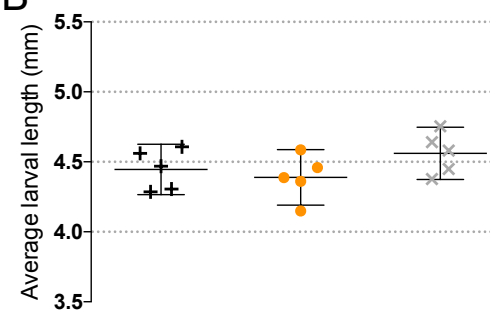

D

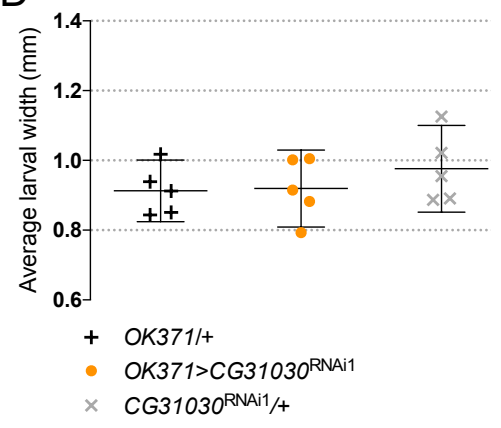

C

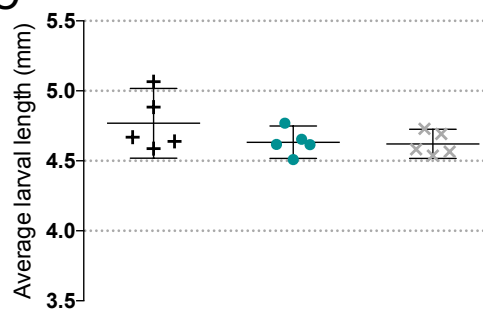

$\mathrm{E}$

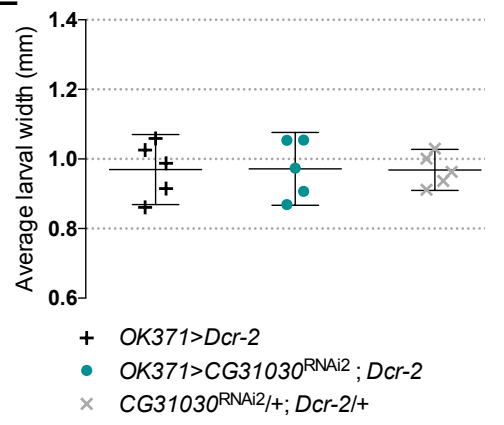

935 Supplementary Figure 3. CG31030 downregulation in motoneurons did not significantly alter larval size.

936 (A) Larval length is defined as the spine length from head to tail, while larval width is the diameter of the

937 mid-spine circle. (B-E) Average larval length (B, C) and width (D, E) were not significantly different

938 between animals expressing CG31030 RNAi in motoneurons and controls. 


\section{Supplementary tables}

945

946 Supplementary Table 1. Pan-neuronal expression of CG31030 rescues the embryonic lethality of

947 CG31030-deficient flies up to the adult stage.

\begin{tabular}{lll}
\hline $\begin{array}{l}\text { Genotype of the progeny flies } \\
\text { 1 }\end{array}$ & $\begin{array}{l}\text { Expected } \\
\text { percentage } \\
\mathbf{2}\end{array}$ & $\begin{array}{l}\text { Scored } \\
\text { percentage } \\
\mathbf{3}\end{array}$ \\
\hline Heterozygous deficiency without CG31030 expression & 40 & 39.7 \\
\hline Heterozygous deficiency with pan-neuronal CG31030 expression & 40 & 53.8 \\
\hline Homozygous deficiency without CG31030 expression & 0 & 0 \\
\hline Homozygous deficiency with pan-neuronal CG31030 expression & 20 & 6.4 \\
\hline
\end{tabular}

$948{ }^{1}$ Rescue of embryonic lethality was assayed by crossing elav-Gal4; CG31030 M1107/TM6B(Tb) females with w;

949 UAS-CG31030/CyO; Df(3R)Exel6214/TM6B( $\underline{T b})$ males, and scoring the relative number of CG31030-expressing

950 adult flies homozygous for CG31030 deficiency (i.e. non-Tb and non-Cy elav-Gal4/+; UAS-CG31030/+;

$951 C G 31030^{\mathrm{MI} 107} / D f(3 R)$ Exel6214) in the progeny (highlighted line).

$952{ }^{2}$ Expected percentage of adult progeny flies of each genotype in case of full rescue.

$953{ }^{3}$ Actual percentage obtained in the experiments. 24 rescued adults were recovered out of a total of 373

954 progeny flies.

955

956

957

958

959

960 
961 Supplementary Table 2. List of the genes encoding proteins that co-immunoprecipitated with CG31030

962 in Drosophila head extracts.

\begin{tabular}{|c|c|c|c|c|}
\hline Gene Symbol $^{1}$ & FlyBase Gene ID & $\log _{2}\left(R_{1}\right)$ & $\log _{2}\left(R_{2}\right)$ & $\log _{2}\left(R_{3}\right)$ \\
\hline ATP6AP2 & FBgn0037671 & 6.154281057 & 9.481786693 & 5.17436482 \\
\hline Vha100-1 & FBgn0028671 & 1.499248537 & 8.012982519 & 5.43599503 \\
\hline VhaAC39-1 & FBgn0028665 & 2.104797507 & 8.293264658 & 4.86741949 \\
\hline CG31030 & FBgn0051030 & 3.669278876 & 7.960835791 & 5,19465574 \\
\hline$T w d l \beta$ & FBgn0033658 & 1.642952792 & 3.068640973 & 2.35207192 \\
\hline Ccp84Ag & FBgn0004777 & 1.543972087 & 1.326485589 & 1.86392045 \\
\hline CG13627 & FBgn0039217 & 5.738384187 & 4.008318188 & 3.18433108 \\
\hline mfas & FBgn0260745 & 1.596554441 & 3.368384477 & 3.03376685 \\
\hline CG16820 & FBgn0032495 & 4.77051909 & 9.784086057 & 3.60280541 \\
\hline Cpr64Ab & FBgn0035511 & 1.08994458 & 2.758609792 & 2.31103817 \\
\hline CG14752 & FBgn0033307 & 2.21698759 & 3.69458382 & 3.10154816 \\
\hline CG15615 & FBgn0034159 & 2.77101281 & 5.12362053 & 3.39808341 \\
\hline
\end{tabular}

963

$964{ }^{1} 12$ proteins were identified with at least twice higher abundance in $C G 31030^{\mathrm{V} 5}$ compared to the $w^{1118}$

965 control in three independent co-immunoprecipitations with anti-V5 antibodies, followed by mass-

966 spectrometry analysis experiments. Among these proteins, three are known to be constitutive or

967 accessory subunits of the V-ATPase complex. They are indicated in the first ochre-highlighted lines of the

968 Table and circled in blue in Fig. 2. The grey line indicates the co-immunoprecipitation target CG31030. 
973 Supplementary Table 3. Drosophila strains used in this study.

974

\begin{tabular}{|c|c|c|c|c|}
\hline Names & Genotypes & Usage & Sources & References \\
\hline$w^{1118}$ & $w^{1118}$ & $\begin{array}{l}\text { Wild-type control } \\
\text { line }\end{array}$ & $\begin{array}{l}\text { In-house } \\
\text { collection }\end{array}$ & Hazelrigg et al. 1984 \\
\hline UAS-CG31030 & $\mathrm{W} ; \mathrm{P}\left\{w^{+\mathrm{mW} \cdot \mathrm{hs}}=C \mathrm{CG} 31030^{\mathrm{UAS}}\right\} ;+$ & $\begin{array}{l}\text { Expression of } \\
\text { Drosophila } \\
\text { CG31030 }\end{array}$ & $\begin{array}{l}\text { In-house } \\
\text { construct }\end{array}$ & This report \\
\hline CG31030 V5 & w; CG31030 v5 & $\begin{array}{l}\text { Insertion of a V5 } \\
\text { tag in } C G 31030\end{array}$ & $\mid \begin{array}{l}\text { In-house } \\
\text { construct }\end{array}$ & This report \\
\hline CG31030 RNAi1 & $\begin{array}{l}y, s c, v, \operatorname{sev}^{21} ; P\left\{y^{+t 7.7}\right. \\
v^{+11.8}=\text { TRiP.HMC05568\}attP4O }\end{array}$ & $\begin{array}{l}\text { CG31030 knock } \\
\text { down }\end{array}$ & $\begin{array}{l}\text { BDSC line } \\
n^{\circ} 64549\end{array}$ & Perkins et al., 2015 \\
\hline CG31030 RNAi2 & $y, w^{1118} ; P\{K K 106825\}$ VIE-260B & $\begin{array}{l}\text { CG31030 knock } \\
\text { down }\end{array}$ & VDRC n¹07398 & Dietzl et al., 2007 \\
\hline CG31030 RNAi3 & $w^{1118} ; P\{G D 381\} v 33095 / \mathrm{TM} 3$ & $\begin{array}{l}\text { CG31030 knock } \\
\text { down }\end{array}$ & VDRC n³3095 & Dietzl et al., 2007 \\
\hline CG31030 & $\begin{array}{l}y, w ; \\
\text { Mif }\left\{y^{+m D i n t 2}=M I C\right\} C G 31030^{M 100107} \\
/ T M 3, \text { Sb, Ser }\end{array}$ & $\begin{array}{l}\text { MIMIC CG31030 } \\
\text { mutant }\end{array}$ & $\begin{array}{l}\text { BDSC line } \\
n^{\circ} 30620\end{array}$ & $\begin{array}{l}\text { Nagarkar-Jaiswal et } \\
\text { al., } 2015\end{array}$ \\
\hline$D f(3 R)$ Exel6214 & $\begin{array}{l}W^{1118} ; D f(3 R) \text { Exel6214, } \\
P\left\{W^{+m C}=X P-U\right\} \text { Exel6214/TM6B, } \\
T b\end{array}$ & $\begin{array}{l}\text { Deficiency covering } \\
\text { CG31030 and } \sim 20 \\
\text { other genes }\end{array}$ & BDSC line $n^{\circ} 7692$ & Parks et al., 2004 \\
\hline elav-Gal4 & $P\left\{w^{+m W \cdot h s}=G a w B\right\} e l a v^{C 155}$ & Pan-neuronal driver & BDSC line $n^{\circ} 458$ & $\begin{array}{l}\text { Lin and Goodman, } \\
1994\end{array}$ \\
\hline OK371-Gal4 & $\begin{array}{l}w^{1118} ; \\
P\left\{w^{\text {mW.hs }^{\prime m}}=\text { GawB }\right\} \text { VGlut }{ }^{\mathrm{OK} 371}\end{array}$ & $\begin{array}{l}\text { Glutamatergic and } \\
\text { motor neuron } \\
\text { driver }\end{array}$ & $\begin{array}{l}\text { Gift of Hermann } \\
\text { Aberle, Heinrich } \\
\text { Heine University, } \\
\text { Düsseldorf, } \\
\text { Germany }\end{array}$ & $\begin{array}{l}\text { Mahr and Aberle, } \\
2006\end{array}$ \\
\hline $\begin{array}{l}\text { UAS-VMAT- } \\
\text { pHluorin }\end{array}$ & $w ; V_{m a t} t^{\text {UAS.pHluorin }}$ & $\begin{array}{l}\text { Expression of } \\
\text { Drosophila Vmat } \\
\text { fused to the } \mathrm{pH}- \\
\text { sensitive } \\
\text { fluorescent marker } \\
\text { ecliptic pHluorin }\end{array}$ & $\begin{array}{l}\text { Gift of David } \\
\text { Krantz,UCLA, Los } \\
\text { Angeles, USA }\end{array}$ & Wu et al., 2013 \\
\hline
\end{tabular}

975

976 


\section{Supplementary methods}

979 Detailed LC-MS/MS procedure

980 Proteins on beads were digested overnight at $37^{\circ} \mathrm{C}$ with trypsin (Promega, Madison, WI, USA) in

$98125 \mathrm{mM} \mathrm{NH}_{4} \mathrm{HCO}_{3}$ buffer $(0.2 \mu \mathrm{g}$ trypsin in $20 \mu \mathrm{L})$. The resulting peptides were desalted using 982 ZipTip $\mu$-C18 Pipette Tips (Pierce Biotechnology, Rockford, IL, USA). Samples were analyzed 983 using either an Orbitrap Fusion or an Orbitrap Q-Exactive Plus, coupled respectively to a Nano-

984 LC Proxeon 1200 or a Nano-LC Proxeon 1000, both equipped with an easy spray ion source 985 (Thermo Fisher Scientific, Waltham, MA, USA). On the Orbitrap Fusion instrument, peptides 986 were loaded with an online preconcentration method and separated by chromatography using a 987 Pepmap-RSLC C18 column (0.75 x 750 mm, $2 \mu \mathrm{m}, 100 \AA ̊$ ) from Thermo Fisher Scientific, 988 equilibrated at $50^{\circ} \mathrm{C}$ and operated at a flow rate of $300 \mathrm{nl} / \mathrm{min}$. Peptides were eluted by a 989 gradient of solvent $A\left(\mathrm{H}_{2} \mathrm{O}, 0.1 \% \mathrm{FA}\right)$ and solvent $\mathrm{B}\left(\mathrm{ACN} / \mathrm{H}_{2} \mathrm{O} 80 / 20,0.1 \% \mathrm{FA}\right)$. The column was 990 first equilibrated for 5 min with $95 \%$ of $A$, then B was raised to $28 \%$ in 105 min and to $40 \%$ in $99115 \mathrm{~min}$. Finally, the column was washed with 95\% B during 20 min and re-equilibrated at $95 \% \mathrm{~A}$ 992 for $10 \mathrm{~min}$. Peptides masses were analyzed in the Orbitrap cell in full ion scan mode, at a 993 resolution of 120,000 , a mass range of $m / z 350-1550$ and an AGC target of $4.10^{5}$. MS/MS were 994 performed in the top speed 3s mode. Peptides were selected for fragmentation by Higher995 energy C-trap Dissociation (HCD) with a Normalized Collisional Energy of $27 \%$ and a dynamic 996 exclusion of 60 seconds. Fragment masses were measured in an lon trap in the rapid mode, with 997 and an AGC target of $1.10^{4}$. Monocharged peptides and unassigned charge states were excluded 
998 from the MS/MS acquisition. The maximum ion accumulation times were set to $100 \mathrm{~ms}$ for MS

999 and $35 \mathrm{~ms}$ for MS/MS acquisitions respectively.

1000 On the Q-Exactive Plus instrument, peptides were loaded with an online preconcentration

1001 method and separated by chromatography using a Pepmap-RSLC C18 column $(0.75 \times 500 \mathrm{~mm}, 2$

$1002 \mu \mathrm{m}, 100 \AA$ ) from Thermo Scientific, equilibrated at $50^{\circ} \mathrm{C}$ and operated at a flow rate of 300

$1003 \mathrm{nl} / \mathrm{min}$. Peptides were eluted by a gradient of solvent $\mathrm{A}\left(\mathrm{H}_{2} \mathrm{O}, 0.1 \% \mathrm{FA}\right)$ and solvent $\mathrm{B}(100 \%$

$1004 \mathrm{ACN}, 0.1 \% \mathrm{FA}$ ), the column was first equilibrated 5 min with $95 \%$ of $\mathrm{A}$, then $\mathrm{B}$ was raised to 35

$1005 \%$ in 93 min and finally, the column was washed with $80 \%$ B during 10 min and re-equilibrated at

$100695 \%$ A during 10 min. Peptides masses were analyzed in the Orbitrap cell in full ion scan mode

1007 at a resolution of 70,000 with a mass range of $m / z 375-1500$ and an AGC target of $3.10^{6}$. MS/MS

1008 were performed in a Top 20 DDA mode. Peptides were selected for fragmentation by Higher-

1009 energy C-trap Dissociation (HCD) with a Normalized Collisional Energy of 27\%, and a dynamic

1010 exclusion of 30 seconds. Fragment masses were measured in the Orbitrap cell at a resolution of

1011 17,500, with an AGC target of 2.10 . Monocharged peptides and unassigned charge states were

1012 excluded from the MS/MS acquisition. The maximum ion accumulation times were set to $50 \mathrm{~ms}$

1013 for MS and $45 \mathrm{~ms}$ for MS/MS acquisitions respectively.

1014

1015 Raw data were processed on Proteome Discoverer 2.4 with the mascot node (Mascot version

1016 2.5.1), with the Swissprot/TrEMBL protein database release 2019_12 for Drosophila

1017 melanogaster. A maximum of 2 missed cleavages was authorized. Precursor and fragment mass

1018 tolerances were set to respectively $7 \mathrm{ppm}$ and $0.5 \mathrm{Da}$ (Orbitrap Fusion) and to $6 \mathrm{ppm}$ and 0.02

1019 Da (Orbitrap Q-exactive Plus). The following post-translational modifications were included as 
1020 variable: Acetyl (Protein N-term), Oxidation (M), Phosphorylation (STY). Spectra were filtered

1021 using a 1\% FDR (false discovery rate) with the percolator node. Label-free quantification was

1022 done in TOP 3 abundance calculation mode with pairwise ratio based calculation and t-test

1023 (background based) hypothesis test.

1024

\section{Quantal analysis}

1026 The probability for an EPSP to contain $i$ quanta followed a binomial distribution:

$$
\mathrm{P}(i)=C_{n}^{i} \times p^{i} \times(1-p)^{(n-i)}
$$

1028 in which $p$ is the average probability of release, and $n$ is the total number of releasable quanta.

1029 In the frequency distribution of EPSP amplitude, successive peaks represent increasing numbers 1030 of quanta.

1031 In order to predict how these events are distributed between different bins in a histogram, it is 1032 necessary to allow for variations in quantal size. To do this, the largest peaks of the histogram 1033 were fitted to a Gaussian curve scaled in width to have a variance proportional to quantal size, 1034 and scaled in height so that its area corresponded to the predicted number of events (Del

1035 Castillo and Katz, 1954). From the mean $(\mu)$ and standard deviation $(\sigma)$ of the Gaussian curve, 1036 the content $(f)$ of each bin $(y)$ is given by:

$1037 f(y)=\frac{1}{\sqrt{2 \pi} \sigma} e^{-\frac{1}{2}\left(\frac{y-\mu}{\sigma}\right)^{2}}$

1038 The standard deviation $(\sigma)$ of each peak depends on the number of quanta it contains. It was 1039 calculated as follows:

1040

$$
\sigma=i \times \sqrt{\sigma_{0}}
$$


1041 with $i$ the number of quanta in the peak, and $\sigma_{0}$ the standard deviation of a single quanta. The 1042 amplitude of Gaussian distribution for each peak is scaled to the probability $P(i)$ for that peak 1043 (see (1)).

1044 The complete theoretical distribution, allowing for variance and peak overlap was then obtained 1045 by pooling the Gaussians for all peaks. In this way, the theoretical distribution could be 1046 superimposed on the histogram for direct comparison with the data. 